\title{
On the Joint Source-Channel Coding Error Exponent for Discrete Memoryless Systems
}

\author{
Yangfan Zhong, Student Member, IEEE, Fady Alajaji, Senior Member, IEEE, and L. Lorne Campbell, Life Fellow, IEEE
}

\begin{abstract}
We investigate the computation of Csiszár's bounds for the joint source-channel coding (JSCC) error exponent $E_{J}$ of a communication system consisting of a discrete memoryless source and a discrete memoryless channel. We provide equivalent expressions for these bounds and derive explicit formulas for the rates where the bounds are attained. These equivalent representations can be readily computed for arbitrary source-channel pairs via Arimoto's algorithm. When the channel's distribution satisfies a symmetry property, the bounds admit closed-form parametric expressions. We then use our results to provide a systematic comparison between the JSCC error exponent $E_{J}$ and the tandem coding error exponent $E_{T}$, which applies if the source and channel are separately coded. It is shown that $E_{T} \leq E_{J} \leq 2 E_{T}$. We establish conditions for which $E_{J}>E_{T}$ and for which $E_{J}=2 E_{T}$. Numerical examples indicate that $E_{J}$ is close to $2 E_{T}$ for many sourcechannel pairs. This gain translates into a power saving larger than $\mathbf{2} \mathrm{dB}$ for a binary source transmitted over additive white Gaussian noise (AWGN) channels and Rayleigh-fading channels with finite output quantization. Finally, we study the computation of the lossy JSCC error exponent under the Hamming distortion measure.
\end{abstract}

Index Terms-Discrete memoryless sources and channels, error exponent, Fenchel's duality, Hamming distortion measure, joint source-channel coding, random-coding exponent, reliability function, sphere-packing exponent, symmetric channels, tandem source and channel coding.

\section{INTRODUCTION}

$\mathbf{T}$ RADITIONALLY, source and channel coding have been treated independently, resulting in what we call a tandem (or separate) coding system. This is because Shannon in 1948 [45] showed that separate source and channel coding incurs no loss of optimality (in terms of reliable transmissibility) provided that the coding block length goes to infinity. In practical implementations, however, there is a price to pay in delay and complexity, for extremely long block length. To begin, we note that joint source-channel coding (JSCC) might be expected to offer improvements for the combination of a source with significant redundancy and a channel with significant noise, since, for such a system, tandem coding would involve source coding to remove redundancy and then channel coding to insert redundancy. It is a natural conjecture that this is not the most efficient approach

\footnotetext{
Manuscript received October 20, 2004; revised December 15, 2005. This work was supported in part by the Natural Sciences and Engineering Research Council of Canada and the Premier's Research Excellence Award of Ontario. The material in this paper was presented in part at the 22nd Biennial Symposium on Communications, Kingston, ON, Canada, June 2004 and the IEEE International Symposium on Information Theory, Chicago, IL, June/July 2004.

The authors are with the Department of Mathematics and Statistics, Queen's University, Kingston, ON K7L 3N6, Canada (e-mail: yangfan@mast. queensu.ca; fady@mast.queensu.ca; campbell@mast.queensu.ca).

Communicated by A. Lapidoth, Associate Editor for Shannon Theory.

Digital Object Identifier 10.1109/TIT.2006.871608
}

(even if the block length is allowed to grow without bound). Indeed, Shannon [45] made this point as follows:

... However, any redundancy in the source will usually help if it is utilized at the receiving point. In particular, if the source already has a certain redundancy and no attempt is made to eliminate it in matching to the channel, this redundancy will help combat noise. For example, in a noiseless telegraph channel one could save about $50 \%$ in time by proper encoding of the messages. This is not done and most of the redundancy of English remains in the channel symbols. This has the advantage, however, of allowing considerable noise in the channel. A sizable fraction of the letters can be received incorrectly and still reconstructed by the context. In fact this is probably not a bad approximation to the ideal in many cases ...

The study of JSCC dates back to as early as the 1960s. Over the years, many works have introduced JSCC techniques and illustrated (analytically or numerically) their benefits (in terms of both performance improvement and increased robustness to variations in channel noise) over tandem coding for given source and channel conditions and fixed complexity and/or delay constraints. In JSCC systems, the designs of the source and channel codes are either well coordinated or combined into a single step. Examples of (both constructive and theoretical) previous lossless and lossy JSCC investigations include the following:

a) JSCC theorems and the separation principle [6], [10], [15], [20], [23], [26], [28], [29], [32], [51];

b) source codes that are robust against channel errors such as optimal (or suboptimal) quantizer design for noisy channels [4], [9], [21], [22], [25], [33]-[35], [39], [41], [47], [48], [50];

c) channel codes that exploit the source's natural redundancy (if no source coding is applied) or its residual redundancy (if source coding is applied) [3], [27], [38], [44], [58];

d) zero-redundancy channel codes with optimized codeword assignment for the transmission of source encoder indices over noisy channels (e.g., [21], [54]);

e) unequal error protection source and channel codes where the rates of the source and channel codes are adjusted to provide various levels of protection to the source data depending on its level of importance and the channel conditions (e.g., [30], [40]);

f) uncoded source-channel matching where the source is uncoded, directly matched to the channel and optimally decoded (e.g., [2], [24], [46], [53]). 
The above references are far from exhaustive as the field of JSCC has been quite active, particularly over the last 20 years.

In order to learn more about the performance of the best codes as a function of block length, much research has focused on the error exponent or reliability function for source or channel coding (see, e.g., [13], [19], [23], [31], [37], [52]). Roughly speaking, the error exponent $E$ is a number with the property that the probability of decoding error of a good code is approximately $2^{-E n}$ for codes of large block length $n$. Thus, the error exponent can be used to estimate the tradeoff between error probability and block length. In this paper, we use the error exponent as a tool to compare the performance of tandem coding and JSCC. While jointly coding the source and channel offers no advantages over tandem coding in terms of reliable transmissibility of the source over the channel (for the case of memoryless systems as well as the wider class of stationary information stable [15], [28] systems), it is possible that the same error performance can be achieved for smaller block lengths via optimal JSCC coding.

The first quantitative result on error exponents for lossless JSCC was a lower bound on the error exponent derived in 1964 by Gallager [23, pp. 534-535]. This result also indicates that JSCC can lead to a larger exponent than the tandem coding exponent, the exponent resulting from separately performing and concatenating optimal source and channel coding. In 1980, Csiszár [17] established a lower bound (based on the random-coding channel error exponent) and an upper bound for the JSCC error exponent $E_{J}(Q, W, t)$ of a communication system with transmission rate $t$ source symbols/channel symbol and consisting of a discrete memoryless source (DMS) with distribution $Q$ and a discrete memoryless channel (DMC) with transition distribution $W$. He showed that the upper bound, which is expressed as the minimum of the sum of $t e(R / t, Q)$ and $E(R, W)$ over $R$, i.e.,

$$
\min _{R}\left[t e\left(\frac{R}{t}, Q\right)+E(R, W)\right]
$$

where $e(R, Q)$ is the source error exponent [13], [17], [31] and $E(R, W)$ is the channel error exponent [17], [23], [31], is tight if the latter minimum is attained for an $R$ strictly larger than the critical rate of the channel. Another (looser) upper bound to $E_{J}(Q, W, t)$ directly results from (1) by replacing $E(R, W)$ by the sphere-packing channel error exponent. He extended this work in 1982 [18] to obtain a new expurgated lower bound (based on the expurgated channel exponent) for the above system under some conditions, and to deal with lossy coding relative to a distortion threshold. Our first objective in this work is to recast Csiszár's results in a form more suitable for computation and to examine the connection between Csiszár's upper and lower bounds. After this, we go on to compare the joint coding and tandem coding error exponents in order to discover how much potential for improvement there is via JSCC. Since error exponents give only asymptotic expressions for system performance, our results do not have direct application to the construction of good codes. Rather, they point out certain systems for which a search for good joint codes might prove fruitful.

We first investigate the analytical computation of Csiszár's random-coding lower bound and sphere-packing upper bound for the JSCC error exponent. By applying Fenchel's Duality Theorem [36] regarding the optimization of the sum of two convex functions, we provide equivalent expressions for these bounds which involve a maximization over a nonnegative parameter of the difference between the concave hull of Gallager's channel function and Gallager's source function [23]; hence, they can be readily computed for arbitrary source-channel pairs by applying Arimoto's algorithm [8]. When the distribution of the channel is symmetric [23], our bounds admit closed-form parametric expressions. We also provide formulas for the rates for which the bounds are attained and establish explicit computable conditions in terms of $Q$ and $W$ under which the upper and lower bounds coincide; in this case, $E_{J}$ can be determined exactly. A byproduct of our results is the observation that Csiszár's JSCC random-coding lower bound can be larger than Gallager's earlier lower bound obtained in [23].

We next employ our results to provide a systematic comparison of the JSCC exponent $E_{J}(Q, W, t)$ and the tandem coding exponent $E_{T}(Q, W, t)$ for a DMS-DMC pair $(Q, W)$ with the same transmission rate $t$. Since $E_{J} \geq E_{T}$ in general (as tandem coding is a special case of JSCC), we are particularly interested in investigating the situation where $E_{J}>E_{T}$. Indeed, this inequality, when it holds, provides a theoretical underpinning and justification for JSCC design as opposed to the widely used tandem approach, since the former method will yield a faster exponential rate of decay for the error probability, which may translate into substantial reductions in complexity and delay for real-world communication systems. We establish sufficient (computable) conditions for which $E_{J}>E_{T}$ for any given source-channel pair $(Q, W)$, which are satisfied for a large class of memoryless source-channel pairs. Furthermore, we show that $E_{J} \leq 2 E_{T}$. Numerical examples show that $E_{J}$ can be nearly twice as large as $E_{T}$ for many DMS-DMC pairs. Thus, for the same error probability, JSCC would require around half the delay of tandem coding. This potential benefit translates into more than 2-dB power gain for binary DMS sent over binary-input quantized-output additive white Gaussian noise and memoryless Rayleigh-fading channels.

We also partially address the computation of Csiszár's lower and upper bounds for the lossy JSCC exponent with distortion threshold $\Delta, E_{J}^{\Delta}(Q, W, t)$. Under the case of the Hamming distortion measure, and for a binary DMS and an arbitrary DMC, we express the bounds for $E_{\bar{J}}^{\Delta}(Q, W, t)$ and the rates for which the bounds are attained as in the lossless case.

The rest of this paper is arranged as follows. In Section II, we describe the system, define the terminologies, and introduce some material on convexity and Fenchel duality. Section III is devoted to study the analytical computation of $E_{J}$ based on Csiszár's work [17], [18]. In Section IV, we assess the merits of JSCC by comparing $E_{J}$ with $E_{T}$. The computation of the lossy JSCC exponent is partially studied in Section V. Finally, we state our conclusions in Section VI. 


\section{DEFINITIONS AND SYSTEM DESCRIPTION}

\section{A. System}

We consider throughout this paper a communication system consisting of a DMS $\{Q: \mathcal{S}\}$ with finite alphabet $\mathcal{S}$ and distribution $Q$, and a DMC $\{W: \mathcal{X} \rightarrow \mathcal{Y}\}$ with finite input alphabet $\mathcal{X}$, finite output alphabet $\mathcal{Y}$, and transition probability $W \triangleq P_{Y \mid X}$. Without loss of generality, we assume that $Q(s)>$ 0 for each $s \in \mathcal{S}$. Also, if the source distribution is uniform, optimal (lossless) JSCC amounts to optimal channel coding which has already been well studied. Therefore, we assume throughout that $Q$ is not the uniform distribution on $\mathcal{S}$ except in Section $\mathrm{V}$, where we deal with JSCC under a fidelity criterion.

A joint source-channel (JSC) code with block length $n$ and transmission rate $t>0$ (measured in source symbols/channel use) is a pair of mappings $f_{n}: \mathcal{S}^{t n} \longrightarrow \mathcal{X}^{n}$ and $\varphi_{n}: \mathcal{Y}^{n} \longrightarrow$ $\mathcal{S}^{t n}$. That is, blocks $s^{t n} \triangleq\left(s_{1}, s_{2}, \ldots, s_{t n}\right)$ of source symbols of length $t n$ are encoded as blocks $x^{n} \triangleq\left(x_{1}, x_{2}, \ldots, x_{n}\right)=$ $f_{n}\left(s^{t n}\right)$ of symbols from $\mathcal{X}$ of length $n$, transmitted, received as blocks $y^{n} \triangleq\left(y_{1}, y_{2}, \ldots, y_{n}\right)$ of symbols from $\mathcal{Y}$ of length $n$, and decoded as blocks of source symbols $\varphi_{n}\left(y^{n}\right)$ of length $t n$. The probability of erroneously decoding the block is

$$
\begin{aligned}
& P_{e}^{(n)}(Q, W, t) \\
& \triangleq \sum_{\left\{\left(s^{t n}, y^{n}\right): \varphi_{n}\left(y^{n}\right) \neq s^{t n}\right\}} Q_{t n}\left(s^{t n}\right) P_{n, Y \mid X}\left(y^{n} \mid f_{n}\left(s^{t n}\right)\right) .
\end{aligned}
$$

Here, $Q_{t n}$ and $P_{n, Y \mid X}$ are the $t n$ - and $n$-dimensional product distributions corresponding to $Q$ and $P_{Y \mid X}$, respectively.

Throughout the paper, $\log$ will denote a base $2 \log$ arithm, $|\mathcal{S}|$ will mean the number of elements in $\mathcal{S}$ and similarly for the other alphabets, and $C$ will denote the capacity of the DMC given by

$$
C=\max _{P_{X}} I\left(P_{X} ; W\right)
$$

where $I\left(P_{X} ; W\right)$ is the mutual information between the channel input and the channel output [23]. Finally, $H(\cdot)$ will denote the entropy of a discrete probability distribution.

\section{B. Error Exponents}

Definition 1: The JSCC error exponent $E_{J}(Q, W, t)$ is defined as the largest number $E$ for which there exists a sequence of JSC codes $\left(f_{n}, \varphi_{n}\right)$ with transmission rate $t$ and block length $n$ such that

$$
E \leq \liminf _{n \rightarrow \infty}-\frac{1}{n} \log P_{e}^{(n)}(Q, W, t)
$$

When there is no possibility of confusion, $E_{J}(Q, W, t)$ will be written as $E_{J}$. We know from the JSCC theorem (e.g., [16, p. 216], [23]) that $E_{J}$ can be positive if and only if $t H(Q)<C$.

For future use, we recall the source and channel functions used by Gallager [23] in his treatment of the JSCC theorem. We also introduce some useful notation and some elementary relations among these functions. Let Gallager's source function be

$$
E_{s}(\rho, Q) \triangleq(1+\rho) \log \sum_{s \in \mathcal{S}} Q(s)^{\frac{1}{1+\rho}}, \quad \rho \geq 0
$$

Let

$$
\tilde{E}_{0}\left(\rho, P_{X}, W\right) \triangleq-\log \sum_{y \in \mathcal{Y}}\left(\sum_{x \in \mathcal{X}} P_{X}(x) P_{Y \mid X}^{\frac{1}{1+\rho}}(y \mid x)\right)^{1+\rho}
$$

where $P_{X}$ is an unspecified probability distribution on $\mathcal{X}$. Connected with these functions are the source error exponent

$$
e(R, Q)=\sup _{0 \leq \rho<\infty}\left[\rho R-E_{s}(\rho, Q)\right]
$$

and two intermediate channel error exponents

$$
\tilde{E}_{r}\left(R, P_{X}, W\right) \triangleq \max _{0 \leq \rho \leq 1}\left[\tilde{E}_{0}\left(\rho, P_{X}, W\right)-\rho R\right]
$$

and

$$
\tilde{E}_{s p}\left(R, P_{X}, W\right) \triangleq \sup _{0 \leq \rho<\infty}\left[\tilde{E}_{0}\left(\rho, P_{X}, W\right)-\rho R\right]
$$

From these, we can form the random-coding lower bound for the channel error exponent $E(R, W)$

$$
E_{r}(R, W) \triangleq \max _{P_{X}} \tilde{E}_{r}\left(R, P_{X}, W\right)
$$

and the sphere-packing upper bound

$$
E_{s p}(R, W) \triangleq \max _{P_{X}} \tilde{E}_{s p}\left(R, P_{X}, W\right)
$$

In other words, $E_{r}(R, W) \leq E(R, W) \leq E_{s p}(R, W)$. Also, we can form Gallager's channel function

$$
E_{0}(\rho, W) \triangleq \max _{P_{X}} \tilde{E}_{0}\left(\rho, P_{X}, W\right) .
$$

It should be noted that maximization over $P_{X}$ means maximization over the closed bounded set

$$
\left\{\left(p_{1}, \ldots, p_{|\mathcal{X}|}\right): p_{i} \geq 0, \sum p_{i}=1\right\}
$$

Thus, if the function involved is continuous, the maximum is achieved for some distribution $\bar{P}_{X}$.

The functions $\tilde{E}_{r}\left(R, P_{X}, W\right)$ and $\tilde{E}_{s p}\left(R, P_{X}, W\right)$ in (5) and (6) are equal if the maximizing $\rho \leq 1$ in (6) or equivalently, if $R \geq R_{c r}\left(P_{X}, W\right)$, where $R_{c r}\left(P_{X}, W\right)$ is the critical rate of the channel $W$ under distribution $P_{X}$, defined by

$$
\left.R_{c r}\left(P_{X}, W\right) \triangleq \frac{\partial \tilde{E}_{0}\left(\rho, P_{X}, W\right)}{\partial \rho}\right|_{\rho=1} .
$$

For all $P_{X}, \tilde{E}_{r}\left(R, P_{X}, W\right)$ and $\tilde{E}_{s p}\left(R, P_{X}, W\right)$ vanish for all $R \geq C$. Consequently, their maxima over $P_{X}, E_{r}(R, W)$, and 
$E_{s p}(R, W)$ vanish for $R \geq C$ and are equal on some interval $\left[R_{c r}(W), C\right]$ where $R_{c r}(W)$ is the critical rate of the channel and is defined by

$$
R_{c r}(W) \triangleq \inf \left\{R: E_{r}(R, W)=E_{s p}(R, W)\right\} .
$$

Furthermore, it is known that $E_{s p}(R, W)$ meets $E_{r}(R, W)$ on its supporting line of slope $-1[19$, p. 171], which means that $E_{r}(R, W)$ is a straight line with slope -1 for $R \leq R_{c r}(W)$ and hence,

$$
E_{r}(R, W)=E_{0}(1, W)-R, \quad R \leq R_{c r}(W) .
$$

We remark that Csiszár [17] defines $e(R, Q), \tilde{E}_{r}\left(R, P_{X}, W\right)$, and $\tilde{E}_{s p}\left(R, P_{X}, W\right)$ using expressions involving constrained minima of Kullback-Leibler divergences. Our expressions are equivalent, as can be shown by the Lagrange multiplier method; see also [19, pp. 192-193] and [13].

\section{Tilted Distributions}

We associate with the source distribution $Q$ a family of tilted distributions $Q^{(\rho)}$ defined by

$$
Q^{(\rho)}(s) \triangleq \frac{Q^{\frac{1}{1+\rho}}(s)}{\sum_{s^{\prime} \in \mathcal{S}} Q^{\frac{1}{1+\rho}}\left(s^{\prime}\right)}, \quad s \in \mathcal{S}, \rho \geq 0 .
$$

Lemma $1[19, p .44]$ : The entropy $H\left(Q^{(\rho)}\right)$ is a strictly increasing function of $\rho$ except in the case that $Q(s)=1 /|\mathcal{S}|$ for all $s \in \mathcal{S}$. Moreover, for $H(Q) \leq R \leq \log |\mathcal{S}|$, the equation $H\left(Q^{(\rho)}\right)=R$ is satisfied by a unique value $\rho^{*}$ (where we define $\rho^{*} \triangleq \infty$ if $R=\log |\mathcal{S}|$ and define $\left.H\left(Q^{(\infty)}\right) \triangleq \log |\mathcal{S}|\right)$.

The proof that $H\left(Q^{(\rho)}\right)$ is increasing follows easily from differentiation with respect to $\rho$ and a use of the Cauchy-Schwarz inequality. The remainder of the proof follows from the facts that $H\left(Q^{(0)}\right)=H(Q), \lim _{\rho \rightarrow \infty} H\left(Q^{(\rho)}\right)=\log |\mathcal{S}|$, and that $H\left(Q^{(\rho)}\right)$ is a continuous function of $\rho$.

It is easily seen that

$$
H\left(Q^{(\rho)}\right)=\frac{\partial E_{s}(\rho, Q)}{\partial \rho}
$$

where $E_{s}(\rho, Q)$ is defined by (2). From this we see that for $R \geq H(Q)$ the supremum in (4) is achieved at $\rho^{*}$.

\section{Fenchel Duality}

Although many of our results can be obtained by the use of the Lagrange multiplier method, the Fenchel Duality Theorem gives more succinct proofs and seems particularly well adapted to the elucidation of the connection between error exponents on the one hand, and source and channel functions on the other. ${ }^{1}$ We present here a simplified one-dimensional version which is adequate for our purposes. For more detailed discussion, the reader may consult [36, pp. 190-202], [12, Ch. 7], or [42].

\footnotetext{
${ }^{1}$ Another related application of Fenchel duality is carried out in [5] in the context of guessing subject to distortion, where it is shown that the guessing exponent is the Fenchel transform of the error exponent for source coding with a fidelity criterion.
}

For any function $f$ defined on $F \subset \mathbb{R}$, define its convex Fenchel transform (conjugate function, Legendre transform) $f^{*}$ by

$$
f^{*}(y) \triangleq \sup _{x \in F}[x y-f(x)]
$$

and let $F^{*}$ be the set $\left\{y: f^{*}(y)<\infty\right\}$. It is easy to see from its definition that $f^{*}$ is a convex function on $F^{*}$. Moreover, if $f$ is convex and continuous, then $\left(f^{*}\right)^{*}=f$. More generally, $f^{* *} \leq f$ and $f^{* *}$ is the convex hull of $f$, i.e., the largest convex function that is bounded above by $f[42$, Sec. 3], [12, Sec. 7.1].

Similarly, for any function $g$ defined on $G \subset \mathbb{R}$, define its concave Fenchel transform $g_{*}$ by

$$
g_{*}(y) \triangleq \inf _{x \in G}[x y-g(x)]
$$

and let $G_{*}$ be the set $\left\{y: g_{*}(y)>-\infty\right\}$. It is easy to see from its definition that $g_{*}$ is a concave function on $G_{*}$. Moreover, if $g$ is concave and continuous, then $\left(g_{*}\right)_{*}=g$. More generally, $g_{* *} \geq g$ and $g_{* *}$ is the concave hull of $g$, i.e., the smallest concave function that is bounded below by $g$.

Fenchel Duality Theorem [36, p. 201]: Assume that $f$ and $g$ are, respectively, convex and concave functions on the nonempty intervals $F$ and $G$ in $\mathbb{R}$ and assume that $F \cap G$ has interior points. Suppose further that $\mu=\inf _{x \in F \cap G}[f(x)-g(x)]$ is finite. Then

$$
\mu=\inf _{x \in F \cap G}[f(x)-g(x)]=\max _{y \in F^{*} \cap G_{*}}\left[g_{*}(y)-f^{*}(y)\right]
$$

where the maximum on the right-hand side is achieved by some $y_{0} \in F^{*} \cap G_{*}$. If the infimum on the left-hand side is achieved by some $x_{0} \in F \cap G$, then

$$
\max _{x \in F}\left[x y_{0}-f(x)\right]=x_{0} y_{0}-f\left(x_{0}\right)
$$

and

$$
\min _{x \in G}\left[x y_{0}-g(x)\right]=x_{0} y_{0}-g\left(x_{0}\right) .
$$

\section{E. Properties of the Source and Channel Functions}

Lemma 2: The source function $E_{s}(\rho, Q)$ defined by (2) is a strictly convex function of $\rho$.

Convexity follows directly from (14) and Lemma 1 . Strict convexity is a consequence of our general assumption that $Q$ is not the uniform distribution. It will be seen from (4) that $e(R, Q)$ is the convex Fenchel transform of $E_{s}(\rho, Q)$. In fact, it is easily checked that (e.g., cf. [19, pp. 44-45])

$$
e(R, Q)= \begin{cases}0, & \text { if } R \leq H(Q) \\ D\left(Q^{\left(\rho^{*}\right)} \| Q\right), & \text { if } H(Q) \leq R \leq \log |\mathcal{S}| \\ \infty, & \text { if } R>\log |\mathcal{S}|\end{cases}
$$

where $D(\cdot \| \cdot)$ denotes the Kullback-Leibler divergence and $\rho^{*}$ is the solution of $H\left(Q^{(\rho)}\right)=R$. Note that (18) implies that $e(R, Q)$ is strictly convex in $R$ on $[H(Q), \log |\mathcal{S}|]$ when the source is nonuniform; otherwise, $H(Q)=\log |\mathcal{S}|$.

The relation between the Gallager's channel function $E_{0}(\rho, W)$ and the random-coding and sphere-packing bounds 
is more complicated. First of all, recall that for each $P_{X}$, $\tilde{E}_{r}\left(R, P_{X}, W\right)$ as defined in (5) is a convex nonincreasing function for all $R$, and is a linear function of $R$ with slope -1 for $R \leq R_{c r}\left(P_{X}, W\right)$ [23, p. 143 ]. It will be convenient to regard this linear function as defining $\tilde{E}_{r}\left(R, P_{X}, W\right)$ for all negative $R$. The random coding bound $E_{r}(R, W)$, which is the maximum of this family of convex functions, is a convex strictly decreasing function of $R$ for $R<C$, and is a linear function of $R$ with slope -1 for all $R$ below the critical rate $R_{c r}(W)$. For $R \geq C, E_{r}(R, W)=0$. Since $E_{r}(R, W)$ is convex, then $-E_{r}(R, W)$ is concave. Let $T_{r}(\rho, W)$ be the concave transform of $-E_{r}(R, W)$, i.e.,

$$
T_{r}(\rho, W) \triangleq \inf _{R \in \mathbb{R}}\left[\rho R+E_{r}(R, W)\right]
$$

It follows from the properties of $E_{r}(R, W)$ noted above that $T_{r}(\rho, W)=-\infty$ for $\rho<0$ and $\rho>1$ and that $T_{r}(\rho, W)$ is finite for $\rho \in[0,1]$.

Lemma 3: The function $T_{r}(\rho, W)$ defined by (19) is the concave hull on the interval $[0,1]$ of the channel function $E_{0}(\rho, W)$ defined in (9). Thus, $E_{0}(\rho, W) \leq T_{r}(\rho, W)$ for $0 \leq \rho \leq 1$.

Proof: We form the concave transform of $E_{0}(R, W)$ on the interval $[0,1]$ to get

$$
\begin{aligned}
\left(E_{0}(\rho, W)\right)_{*} & =\inf _{0 \leq \rho \leq 1}\left[\rho R-E_{0}(\rho, W)\right] \\
& =-\sup _{0 \leq \rho \leq 1}\left[E_{0}(\rho, W)-\rho R\right] .
\end{aligned}
$$

Now use, in succession, (9), (5), and (7) to get

$$
\begin{aligned}
\left(E_{0}(\rho, W)\right)_{*} & =-\sup _{0 \leq \rho \leq 1} \max _{P_{X}}\left[\tilde{E}_{0}\left(\rho, P_{X}, W\right)-\rho R\right] \\
& =-\max _{P_{X}} \sup _{0 \leq \rho \leq 1}\left[\tilde{E}_{0}\left(\rho, P_{X}, W\right)-\rho R\right] \\
& =-\max _{P_{X}} \tilde{E}_{r}\left(R, P_{X}, W\right) \\
& =-E_{r}(R, W) .
\end{aligned}
$$

Since $T_{r}(\rho, W)$ is the concave transform of the concave function, $-E_{r}(R, W)$, we have that

$$
\left(-E_{r}(R, W)\right)_{*}=T_{r}(\rho, W) \text { and so }\left(E_{0}(\rho, W)\right)_{* *}=T_{r}(\rho, W) .
$$

Hence, $T_{r}(\rho, W)$ is the concave hull on $[0,1]$ of $E_{0}(\rho, R)$.

Similarly to the above, recall that $E_{s p}(R, W)$, defined in (8) is convex, zero for $R \geq C$, positive for $R<C$, and finite if $R>R_{\infty}(W)$ [19], [23], where $R_{\infty}(W)$ is given by

$$
R_{\infty}(W) \triangleq \lim _{\rho \rightarrow \infty} \frac{E_{0}(\rho, W)}{\rho} .
$$

A computable expression for $R_{\infty}(W)$ is given in [23, p. 158]. The normal situation is $R_{\infty}(W)=0$. (As shown by Gallager, $R_{\infty}(W)=0$ unless each channel output symbol is unreachable from at least one input. In the latter case, $R_{\infty}(W)>0$.) We now let $T_{s p}(\rho, W)$ be the concave transform of the concave function $-E_{s p}(R, W)$, i.e.,

$$
T_{s p}(\rho, W) \triangleq \inf _{R_{\infty}(W)<R<\infty}\left[\rho R+E_{s p}(R, W)\right] .
$$

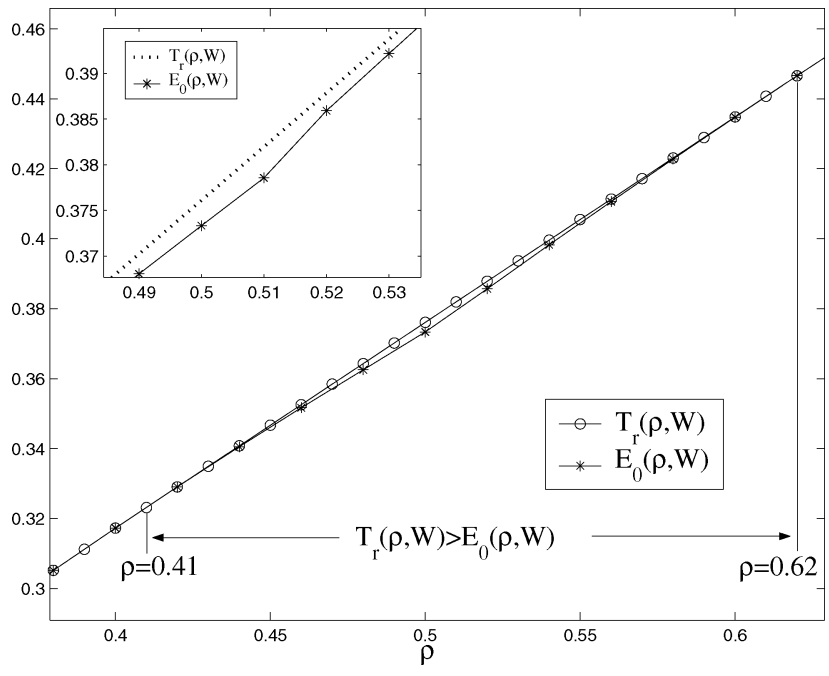

Fig. 1. Example of a 6-ary input, 4-ary output DMC (see [23, Fig. 5.6.5]) for which $E_{0}(\rho, W)$ is not concave.

It follows that $T_{s p}(\rho, W)=-\infty$ for $\rho<0$ and that $0 \leq$ $T_{s p}(\rho, W)<\infty$ for $\rho \geq 0$.

Lemma 4: The function $T_{s p}(\rho, W)$ defined by (21) is the concave hull on $[0, \infty)$ of the channel function $E_{0}(\rho, W)$ defined in (9).

Proof: We now form the concave transform of $E_{0}(\rho, W)$ on the interval $[0, \infty)$ to get

$$
\begin{aligned}
\left(E_{0}(\rho, W)\right)_{*} & =\inf _{0 \leq \rho<\infty}\left[\rho R-E_{0}(\rho, W)\right] \\
& =-\sup _{0 \leq \rho<\infty}\left[E_{0}(\rho, W)-\rho R\right] .
\end{aligned}
$$

Now use (9), (6), and (8) to get

$$
\begin{aligned}
\left(E_{0}(\rho, W)\right)_{*} & =-\sup _{0 \leq \rho<\infty} \max _{P_{X}}\left[\tilde{E}_{0}\left(\rho, P_{X}, W\right)-\rho R\right] \\
& =-\max _{P_{X}} \sup _{0 \leq \rho<\infty}\left[\tilde{E}_{0}\left(\rho, P_{X}, W\right)-\rho R\right] \\
& =-\max _{P_{X}} \tilde{E}_{s p}\left(R, P_{X}, W\right) \\
& =-E_{s p}(R, W) .
\end{aligned}
$$

As in the previous proof, $\left(E_{0}(\rho, W)\right)_{* *}=T_{s p}(\rho, W)$. Hence, $T_{s p}(\rho, W)$ is the concave hull on $[0, \infty)$ of $E_{0}(\rho, R)$.

Observation 1: Note that the function $\tilde{E}_{0}\left(\rho, P_{X}, W\right)$ is concave in $\rho$ for each $P_{X}$ [23, p. 142]. Hence, if the maximizing $P_{X}$ in (9) is independent of $\rho, E_{0}(\rho, W)$ is concave and thus, $T_{r}(\rho, W)$ and $T_{s p}(\rho, W)$ are equal to $E_{0}(\rho, W)$. This situation holds if the channel is symmetric in the sense of Gallager [23, p. 94] (also see Example 2). For this case, the maximizing distribution is the uniform distribution $P_{X}(x)=1 /|\mathcal{X}|$ for all $x \in \mathcal{X}$. However, there are channels for which $E_{0}(\rho, W)$ is not concave. One example of such a channel is provided by Gallager [23, Fig. 5.6.5]. For this particular (6-ary input, 4-ary output) channel, we plot $E_{0}(\rho, W)$ against $\rho$ in Fig. 1 . It is noted that the derivative of $E_{0}(\rho, W)$ has a positive jump increase at around $\rho=0.51$ (see [23, Fig. 5.6.5]), and its concave hull $T_{r}(\rho, W)$ is strictly larger than $E_{0}(\rho, W)$ in the interval $\rho \in(0.41,0.62)$. 


\section{BOUNDS ON THE JSCC ERROR EXPONENT}

\section{A. Csiszár's Random-Coding and Sphere-Packing Bounds}

Csiszár [17] proved that for a DMS and a DMC the JSCC error exponent in Definition 1 satisfies

$$
\underline{E}_{r}(Q, W, t) \leq E_{J}(Q, W, t) \leq \bar{E}_{s p}(Q, W, t)
$$

where

$$
\underline{E}_{r}(Q, W, t) \triangleq \min _{t H(Q) \leq R \leq t \log |\mathcal{S}|}\left[t e\left(\frac{R}{t}, Q\right)+E_{r}(R, W)\right]
$$

and

$\bar{E}_{s p}(Q, W, t) \triangleq \inf _{t H(Q) \leq R \leq t \log |\mathcal{S}|}\left[t e\left(\frac{R}{t}, Q\right)+E_{s p}(R, W)\right]$

are called the source-channel random-coding lower bound and the source-channel sphere-packing upper bound, since they respectively contain $E_{r}(R, W)$ and $E_{s p}(R, W)$ in their expressions. These bounds can be expressed in a form more adapted to calculation as follows.

Theorem 1: Let $t H(Q)<C$ and let $t \log |\mathcal{S}|>R_{\infty}(W)$. Then

$$
\underline{E}_{r}(Q, W, t)=\max _{0 \leq \rho \leq 1}\left[T_{r}(\rho, W)-t E_{s}(\rho, Q)\right]
$$

and

$$
\bar{E}_{s p}(Q, W, t)=\max _{0 \leq \rho<\infty}\left[T_{s p}(\rho, W)-t E_{s}(\rho, Q)\right]
$$

where $T_{r}(\rho, W)$ and $T_{s p}(\rho, W)$ are the concave hulls of $E_{0}(\rho, W)$ on $[0,1]$ and $[0, \infty)$ defined in (19) and (21), respectively. If the maximizing $P_{X}$ in (9) is independent of $\rho$, $T_{r}(\rho, W)$ and $T_{s p}(\rho, W)$ can be replaced by $E_{0}(\rho, W)$.

Remark 1: When $t H(Q) \geq C$,

$$
\underline{E}_{r}(Q, W, t)=\bar{E}_{s p}(Q, W, t)=0 .
$$

Observation 2: According to Lemma $3, E_{0}(\rho, W) \leq$ $T_{r}(\rho, W)$. Thus, the lower bound $\underline{E}_{r}(Q, W, t)$ can be replaced by the possibly looser lower bound ${ }^{2}$

$$
\max _{0 \leq \rho \leq 1}\left[E_{0}(\rho, W)-t E_{s}(\rho, Q)\right]
$$

This is the lower bound implied by Gallager's work [23, p. 535]. As noted earlier, if the maximizing $P_{X}$ in (9) is independent of

2In [56], [55], we incorrectly stated that Csiszár's random-coding lower bound $E_{r}(Q, W, t)$ given in (23) and Gallager's lower bound given in (27) are identical. This is indeed not always true; it is true if $E_{0}(\rho, W)$ is a concave function of $\rho$ (e.g., for symmetric channels) or $t H\left(Q^{(1)}\right) \leq R_{c r}(W)$ (see Corollary 2). Thus, although both lower bounds are "random coding" type bounds, Csiszár's bound is in general tighter. $\rho$ (e.g., for symmetric channels, see Example 2), the two lower bounds are identical.

Proof of Theorem 1: We first apply Fenchel's Duality Theorem (15) to the lower bound $\underline{E}_{r}(Q, W, t)$. From Lemma 2, (4), and (18), te $(R / t, Q)$ is convex on $(-\infty, t \log |\mathcal{S}|]$ and has convex transform $t E_{s}(\rho, Q)$ on the set $[0, \infty)$. Also, from the discussion preceding Lemma $3,-E_{r}(R, W)$ is concave on $\mathbb{R}$ and has concave transform $T_{r}(\rho, W)$ which is bounded on $[0,1]$. Thus, by Fenchel's Duality Theorem

$$
\begin{aligned}
\inf _{-\infty \leq R \leq t \log |\mathcal{S}|}\left[t e\left(\frac{R}{t}, Q\right)+E_{r}(R, W)\right] \\
\quad=\max _{0 \leq \rho \leq 1}\left[T_{r}(\rho, W)-t E_{s}(\rho, Q)\right] .
\end{aligned}
$$

Now, the convex function $t e(R / t, Q)+E_{r}(R, W)$ is nonincreasing for $R \leq t H(Q)$ since $t e(R / t, Q)=0$ in this region. This implies that the infimum on the left-hand side of (28) can be restricted to the interval $t H(Q) \leq R \leq t \log |\mathcal{S}|$. Since this is now the infimum of a continuous function on a finite interval this will be a minimum. Hence, (25) is an equivalent representation of $\underline{E}_{r}(Q, W, t)$.

Similarly, for the upper bound, recall from the discussion preceding Lemma 4 that $-E_{s p}(R, W)$ is concave and finite for $R>R_{\infty}(W)$ and has a concave transform $T_{s p}(\rho, W)$, which is finite on $0 \leq \rho<\infty$. Thus, by Fenchel's Duality Theorem

$$
\begin{aligned}
\inf _{\infty}(W)<R \leq t \log |\mathcal{S}| & {[t e} \\
& \left.=\max _{0 \leq \rho<\infty}\left[\frac{R}{t}, Q\right)+E_{s p}(R, W)\right] \\
&
\end{aligned}
$$

The assumption $R_{\infty}(W)<t \log |\mathcal{S}|$ ensures that the infimum on the left-hand side of (29) is taken over a set with interior points. If $R_{\infty}(W)<t H(Q)$, the infimum can be replaced by a minimum on the interval $t H(Q) \leq R \leq t \log |\mathcal{S}|$ by the same argument as for the lower bound. If $R_{\infty}(W) \geq t H(Q)$, we no longer form the infimum of a continuous function, but it can still be shown that there is a minimum point which lies in the interval $t H(Q) \leq R \leq t \log |\mathcal{S}|$. Hence, (29) is an equivalent representation of $\bar{E}_{s p}(Q, W, t)$.

Observation 3: The parametric form of the lower and upper bounds (25) and (26) indeed facilitates the computation of Csiszár's bounds. In order to compute the bounds for general nonsymmetric channels (when $t H(Q)<C$ and $t \log |\mathcal{S}|>R_{\infty}$ ), one could employ Arimoto's algorithm [8] to find the maximizing distribution and thus $E_{0}(\rho, W)$. We then can immediately obtain the concave hulls of $E_{0}(\rho, W), T_{r}(\rho, W)$, and $T_{s p}(\rho, W)$ numerically (e.g., using Matlab) and thus the maxima of $T_{r}(\rho, W)-t E_{s}(\rho, Q)$ and $T_{s p}(\rho, W)-t E_{s}(\rho, Q)$. This significantly reduces the computational complexity since to compute (23) and (24), we need to first compute $E_{r}(R, W)$ and $E_{s p}(R, W)$ for each $R$, which requires almost the same complexity as above, and then we need to find the minima by searching over all $R$ 's. For symmetric channels, (25) and (26) are analytically solved; see Example 2. 


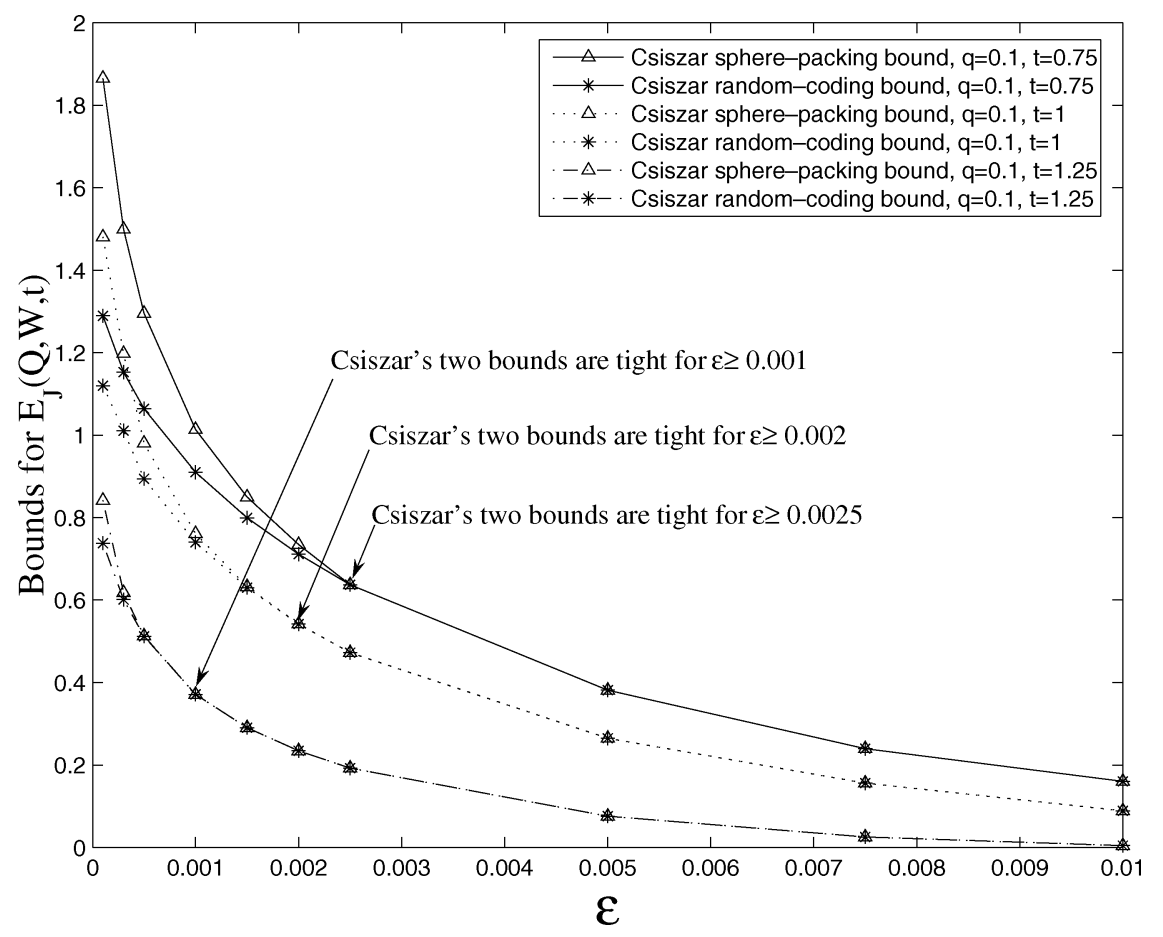

Fig. 2. Csiszár's random-coding and sphere-packing bounds for the system of Example 1.

Example 1: Consider a communication system with a binary DMS with distribution $Q=\{q, 1-q\}$ and a DMC with $|\mathcal{X}|=6$, $|\mathcal{Y}|=4$, and transition probability matrix

$W=\left[\begin{array}{cccc}1-18 \varepsilon & 6 \varepsilon & 6 \varepsilon & 6 \varepsilon \\ 6 \varepsilon & 1-18 \varepsilon & 6 \varepsilon & 6 \varepsilon \\ 6 \varepsilon & 6 \varepsilon & 1-18 \varepsilon & 6 \varepsilon \\ 6 \varepsilon & 6 \varepsilon & 6 \varepsilon & 1-18 \varepsilon \\ 0.5-\varepsilon & 0.5-\varepsilon & \varepsilon & \varepsilon \\ \varepsilon & \varepsilon & 0.5-\varepsilon & 0.5-\varepsilon\end{array}\right], 0 \leq \varepsilon \leq \frac{1}{18}$.

We then compute Csiszár's random-coding and spherepacking bounds, $\underline{E}_{r}(Q, W, t)$ and $\bar{E}_{s p}(Q, W, t)$. For fixed $Q$ and transmission rate $t$, we plot these bounds in terms of $\varepsilon$ in Fig. 2. Our numerical results show that $E_{J}$ could be determined exactly for a large class of $(q, \varepsilon, t)$ triplets: when source $Q=\{0.1,0.9\}$ and rate $t=0.75, E_{J}$ is exactly known for $\varepsilon \geq 0.0025$; when $Q=\{0.1,0.9\}$ and $t=1, E_{J}$ is known for $\varepsilon \geq 0.002$; and when $Q=\{0.2,0.8\}$ and $t=1.25, E_{J}$ is known for $\varepsilon \geq 0.001$. Since for this channel $E_{o}(\rho, W)$ might not be concave (e.g., when $\varepsilon=0.01, W$ reduces to the DMC discussed in Observation 1 at the end of Section II), our results indicate that Csiszár's lower bound is slightly but strictly larger (by $\approx 0.0001$ ) than Gallager's lower bound (27) for $q=0.1$, $t=1$, and $\varepsilon$ around 0.02 (cf. [57, Fig. 3]).

\section{B. When Does $\underline{E}_{r}(Q, W, t)=\bar{E}_{s p}(Q, W, t)$ ?}

One important objective in investigating the bounds for the JSCC error exponent $E_{J}$ is to ascertain when the bounds are tight so that the exact value of $E_{J}$ is obtained. According to Csiszár's result (22), we note that if the minimum in the expressions of $\underline{E}_{r}(Q, W, t)$ or $\bar{E}_{s p}(Q, W, t)$ is attained for a rate (strictly) larger than the critical rate $R_{c r}(W)$, then the two bounds coincide and thus $E_{J}$ is determined exactly. This raises the following question: how can we check whether the minimum in $\underline{E}_{r}(Q, W, t)$ or $\bar{E}_{s p}(Q, W, t)$ is attained for a rate larger than $R_{c r}(W)$ ? One may indeed wonder if there exist explicit conditions for which $\underline{E}_{r}(Q, W, t)=\bar{E}_{s p}(Q, W, t)$. The answer is affirmative; furthermore, we can verify whether the two bounds are tight in two ways: one is to compare $t H\left(Q^{(1)}\right)$ with $R_{c r}(W)$, and the other is to compare the minimizer of $\bar{E}_{s p}(Q, W, t)$ in (26), $\bar{\rho}^{*}$ say, with 1 . Before we present these conditions, we first define the following quantities which achieve the bounds $\underline{E}_{r}(Q, W, t)$ and $\bar{E}_{s p}(Q, W, t)$ under the assumptions $t H(Q)<C$ and $t \log |\mathcal{S}|>R_{\infty}(W)$ :

$$
\begin{aligned}
& \underline{R}_{m} \triangleq \arg \min _{t H(Q) \leq R \leq t \log |\mathcal{S}|}\left[t e\left(\frac{R}{t}, Q\right)+E_{r}(R, W)\right] \\
& \bar{R}_{m} \triangleq \arg \min _{t H(Q) \leq R \leq t \log |\mathcal{S}|}\left[t e\left(\frac{R}{t}, Q\right)+E_{s p}(R, W)\right] \\
& \underline{\rho}^{*} \triangleq \arg \max _{0 \leq \rho \leq 1}\left[T_{r}(\rho, W)-t E_{s}(\rho, Q)\right] \\
& \bar{\rho}^{*} \triangleq \arg \max _{0 \leq \rho<\infty}\left[T_{s p}(\rho, W)-t E_{s}(\rho, Q)\right] .
\end{aligned}
$$

Since the functions between brackets to be minimized (or maximized) in (30)-(33) are strictly convex (or concave) functions of $R$ (or $\rho$ ), $\underline{R}_{m}, \bar{R}_{m}, \rho^{*}$, and $\bar{\rho}^{*}$ are well defined and unique. We then have the following relations.

Lemma 5: Let $t H(Q)<C$ and let $t \log |\mathcal{S}|>R_{\infty}(W)$. Then we have the following:

1) $\bar{\rho}^{*}$ and $\rho^{*}$ are positive and finite;

2) $\bar{R}_{m}=\bar{t} H\left(Q^{\left(\bar{\rho}^{*}\right)}\right)$;

3) $\underline{R}_{m}=t H\left(Q^{\left(\underline{\rho}^{*}\right)}\right)$ if $\underline{\rho}^{*}<1 ; \underline{R}_{m} \geq t H\left(Q^{(1)}\right)$ if $\underline{\rho}^{*}=1$.

Proof: We first prove 1). Since $T_{s p}(\rho, W)$ is the concave hull of $E_{0}(\rho, W)$, we have the following relation:

$$
\lim _{\rho \downarrow 0} \frac{T_{s p}(\rho, W)}{\rho} \geq \lim _{\rho \downarrow 0} \frac{E_{0}(\rho, W)}{\rho}=C
$$


where the last equality follows from [7, Lemma 2]. Since $\lim _{\rho \downarrow 0} E_{s}(\rho, Q) / \rho=H(Q)$ by (14) and Lemma 1, we have

$$
\lim _{\rho \downarrow 0} \frac{T_{s p}(\rho, W)-t E_{s}(\rho, Q)}{\rho} \geq C-t H(Q)>0 .
$$

Note that the right-derivative of $T_{s p}(\rho, W)$ (at $\rho=0$ ) must exist due to its concavity [43, pp. 113-114], and hence $\lim _{\rho \downarrow 0} T_{s p}(\rho, W) / \rho$ exists. Next we denote

$$
\varepsilon=t \log |\mathcal{S}|-R_{\infty}(W)>0 .
$$

It follows from the definition of $T_{s p}(\rho, W)$ that

$$
\begin{aligned}
\lim _{\rho \rightarrow \infty} & \frac{T_{s p}(\rho, W)}{\rho} \\
\leq & \lim _{\rho \rightarrow \infty} \frac{\rho\left(R_{\infty}(W)+\varepsilon / 2\right)+E_{s p}\left(R_{\infty}(W)+\varepsilon / 2, W\right)}{\rho} \\
\quad & R_{\infty}(W)+\varepsilon / 2
\end{aligned}
$$

because of the finiteness of $E_{s p}(R, W)$ for $R>R_{\infty}(W)$. This together with $\lim _{\rho \rightarrow \infty} E_{s}(\rho, Q) / \rho=\log |\mathcal{S}|$ implies

$$
\lim _{\rho \rightarrow \infty} \frac{T_{s p}(\rho, W)-t E_{s}(\rho, Q)}{\rho} \leq R_{\infty}(W)+\varepsilon / 2-t \log |\mathcal{S}|<0 .
$$

Since $T_{s p}(\rho, W)-t E_{s}(\rho, Q)$ is 0 and has a positive right-slope at $\rho=0$ and is negative for $\rho$ sufficiently large, by the strict concavity of $T_{s p}(\rho, W)-t E_{s}(\rho, Q)$, the maximum in (33) must be achieved by a positive finite $\bar{\rho}^{*}$. The positivity of $\rho^{*}$ can be shown in the same way and $\rho^{*}$ is finite by its definition.

We next prove 2). If we now regard $t e(R / t, Q)$ as $f^{*}(y)$ and $t E_{s}(\rho, Q)$ as $f(x)$ (by noting that $f^{* *}=f$ ), then according to (16) in Fenchel's Duality Theorem

$$
\max _{0 \leq \rho<\infty}\left[\rho \bar{R}_{m}-t E_{s}(\rho, Q)\right]=\bar{\rho}^{*} \bar{R}_{m}-t E_{s}\left(\bar{\rho}^{*}, Q\right) .
$$

Setting the derivative of $\rho \bar{R}_{m}-t E_{s}(\rho, Q)$ equal to 0 , we can solve for the stationary point ${ }^{3} \bar{\rho}^{*}$, which gives $\bar{R}_{m}=$ $t H\left(Q^{\left(\bar{\rho}^{*}\right)}\right)$.

For the lower bound, using a similar argument, we obtain the relation

$$
\max _{0 \leq \rho \leq 1}\left[\rho \underline{R}_{m}-t E_{s}(\rho, Q)\right]=\underline{\rho}^{*} \underline{R}_{m}-t E_{s}\left(\underline{\rho}^{*}, Q\right) .
$$

Recalling that the function between the brackets to be maximized is strictly concave, if the above maximum is achieved by $\rho^{*} \in(0,1)$, then we can solve for the stationary point as above and obtain $\underline{R}_{m}=t H\left(Q^{\left(\underline{\rho}^{*}\right)}\right)$. If the maximum is achieved at $\underline{\rho}^{*}=1$, then the stationary point is beyond (at least equal to) 1 , and hence $\underline{R}_{m} \geq t H\left(Q^{(1)}\right)$. Thus (3) follows.

In order to summarize the explicit conditions for the calculation of $E_{J}$, it is convenient to define a critical rate for the source by

$$
\left.R_{c r}^{(s)}(Q) \triangleq \frac{\partial E_{s}(\rho, Q)}{\partial \rho}\right|_{\rho=1}=H\left(Q^{(1)}\right)
$$

recalling that $Q^{(1)}(s)=\sqrt{Q(s)} /\left(\sum_{s^{\prime} \in \mathcal{S}} \sqrt{Q\left(s^{\prime}\right)}\right), s \in \mathcal{S}$.

\footnotetext{
${ }^{3}$ The stationary points of a differentiable function $f(x)$ are the solutions of $f^{\prime}(x)=0$.
}

Theorem 2: Let $t H(Q)<C$ and let $t \log |\mathcal{S}|>R_{\infty}(W)$. Then we have the following.

$$
\begin{aligned}
& t R_{c r}^{(s)}(Q) \geq R_{c r}(W) \\
& \quad \Longleftrightarrow \bar{\rho}^{*} \leq 1 \Longleftrightarrow t R_{c r}^{(s)}(Q) \geq \bar{R}_{m}=\underline{R}_{m} \geq R_{c r}(W) .
\end{aligned}
$$

In this case

$$
E_{J}(Q, W, t)=T_{s p}\left(\bar{\rho}^{*}, W\right)-t E_{s}\left(\bar{\rho}^{*}, Q\right) .
$$

$$
\begin{aligned}
& t R_{c r}^{(s)}(Q)<R_{c r}(W) \\
& \quad \Longleftrightarrow \bar{\rho}^{*}>1 \Longleftrightarrow R_{c r}(W) \geq \bar{R}_{m}>\underline{R}_{m}=t R_{c r}^{(s)}(Q) .
\end{aligned}
$$

In this case,

$$
\begin{aligned}
& E_{0}(1, W)-t E_{s}(1, Q) \\
& \quad \leq E_{J}(Q, W, t) \leq T_{s p}\left(\bar{\rho}^{*}, W\right)-t E_{s}\left(\bar{\rho}^{*}, Q\right) .
\end{aligned}
$$

Remark 2: Under the condition $t R_{c r}^{(s)}(Q)>R_{c r}(W), \bar{\rho}^{*}=$ 1 is possible. However, if $t R_{c r}^{(s)}(Q)=R_{c r}(W)$, then we definitely have $\bar{\rho}^{*}=1$ and $t R_{c r}^{(s)}(Q)=\bar{R}_{m}=\underline{R}_{m}=R_{c r}(W)$.

Remark 3: It can be shown that $T_{s p}(1, W)=E_{0}(1, W)$ and thus, when $\bar{\rho}^{*}=1$, the JSCC exponent is determined by

$$
E_{J}(Q, W, t)=E_{0}(1, W)-t E_{s}(1, Q) .
$$

The proof of Theorem 2 involves a geometric argument involving the left- and right-slopes of the convex functions $E_{r}(R, W)$ and $E_{s p}(R, W)$ and is tedious; we hereby provide the proof when the channel is symmetric for the sake of simplicity. For the full proof (involving nonsymmetric channels), the reader may consult [57, Appendix A].

Proof of Theorem 2 for Symmetric DMCs: When the channel is symmetric, then $E_{0}(\rho, W)$ is concave and differentiable in $\rho \in[0, \infty)$. In this case, it is easy to see by definition that $\underline{\rho}^{*}=\min \left\{1, \bar{\rho}^{*}\right\}$ and $\underline{R}_{m}=t H\left(Q^{\left(\underline{\rho}^{*}\right)}\right)$ since

$$
T_{s p}(\rho, W)=T_{r}(\rho, W)=E_{0}(\rho, W) .
$$

Furthermore, it follows from Lemma 5 and (17) in Fenchel's Duality Theorem (as in the last proof) that

$$
\bar{R}_{m}=t H\left(Q^{\left(\bar{\rho}^{*}\right)}\right)=r\left(\bar{\rho}^{*}\right)
$$

where $r(\rho) \triangleq \partial E_{0}(\rho, W) / \partial \rho$ is decreasing in $\rho([23$, p. 142] $)$ for symmetric DMCs. Now to show $A \Longleftrightarrow B \Longleftrightarrow C$, we need to show: $A \Longrightarrow B$ (Forward) and $B \Longrightarrow C \Longrightarrow A$ (Converse).

1) Converse Part. When $\bar{\rho}^{*} \leq 1$, from the above argument along with Lemma 5 we have

$$
\begin{aligned}
t R_{c r}^{(s)}(Q) \geq \underline{R}_{m} & =\bar{R}_{m}=t H\left(Q^{\left(\bar{\rho}^{*}\right)}\right) \\
& =r\left(\bar{\rho}^{*}\right) \geq r(1)=R_{c r}(W)
\end{aligned}
$$

by the monotonicity of $H\left(Q^{(\rho)}\right)$ and $r(\rho)$. Similarly, when $\bar{\rho}^{*}>1$, it follows from the above argument, 
Lemma 5 and the monotonicity of $H\left(Q^{(\rho)}\right)$ and $r(\rho)$ that

$$
\begin{aligned}
R_{c r}(W) & =r(1) \geq r\left(\bar{\rho}^{*}\right)=\bar{R}_{m} \\
& =t H\left(Q^{\left(\bar{\rho}^{*}\right)}\right)>t R_{c r}^{(s)}(Q)=\underline{R}_{m} .
\end{aligned}
$$

Note that $H\left(Q^{(\rho)}\right)$ is strictly increasing in $\rho$ by Lemma 1.

2) Forward Part. Setting the auxiliary function

$$
\varphi(\rho) \triangleq t H\left(Q^{(\rho)}\right)-r(\rho)
$$

which is strictly increasing in $\rho$ (for $\rho \geq 0$ ), yields via Lemma 5 and the above argument that $\bar{\rho}^{*}$ is the unique root of equation $\varphi(\rho)=0$. To determine the range of $\bar{\rho}^{*}$, we consider the following three cases.

- When $t \log |\mathcal{S}| \geq C$, we consider the interval $[t H(Q), \min (C, t \log |\mathcal{S}|)]=[t H(Q), C]$. It is seen that

$$
\varphi(0)=t H(Q)-r(0)=t H(Q)-C<0
$$

and

$$
\varphi(1)=t H\left(Q^{(1)}\right)-r(1)=t R_{c r}^{(s)}(Q)-R_{c r}(W) .
$$

If $t R_{c r}^{(s)}(Q) \geq R_{c r}(W)$, which means $\varphi(1) \geq 0$, it follows by the strict monotonicity of $\varphi(\cdot)$ that $0<\bar{\rho}^{*} \leq 1$. If $t R_{c r}^{(s)}(Q)<R_{c r}(W)$, then $\varphi(1)<0$, and the monotonicity of $\varphi(\cdot)$ yields $\bar{\rho}^{*}>1$.

- If $R_{c r}(W) \leq t \log |\mathcal{S}| \leq C$, we consider the interval

$$
[t H(Q), \min (C, t \log |\mathcal{S}|)]=[t H(Q), t \log |\mathcal{S}|] .
$$

Since $r(\rho)$ is decreasing in $\rho$, there must exist an $\rho_{0}$ $\left(0 \leq \rho_{0} \leq 1\right)$ such that $r\left(\rho_{0}\right)=t \log |\mathcal{S}|$. It follows that for a nonuniform distribution $Q$

$$
\varphi\left(\rho_{0}\right)=t H\left(Q^{\left(\rho_{0}\right)}\right)-t \log |\mathcal{S}|<0 .
$$

Observing (as above) that $\varphi(1) \geq 0$ if $t R_{c r}^{(s)}(Q) \geq$ $R_{c r}(W)$, it follows that $\rho_{0} \leq \bar{\rho}^{*} \leq 1$. Likewise, if $t R_{c r}^{(s)}(Q)<R_{c r}(W)$, then $\varphi(1)<0$, and we have $\bar{\rho}^{*}>1$.

- If $t \log |\mathcal{S}| \leq R_{c r} \leq C$, then clearly we have

$$
t R_{c r}^{(s)}(Q)<t \log |\mathcal{S}| \leq R_{c r}
$$

since $Q$ is nonuniform. Under this case, we must have $\varphi(1)<0$, which implies that $\bar{\rho}^{*}>1$ by the strict monotonicity of $\varphi(\rho)$.

Corollary 1: Let $t H(Q)<C$ and let $t \log |\mathcal{S}|>R_{\infty}(W)$. Then $\underline{\rho}^{*}=\min \left\{1, \bar{\rho}^{*}\right\}$ and $\underline{R}_{m}=t H\left(Q^{\left(\underline{\rho}^{*}\right)}\right)$.

For symmetric DMCs, Corollary 1 is trivial and has been shown in the last proof. For general DMCs, the proof of Corollary 1 is provided in [57, Appendix A].

We point out that, in both the computation and analysis aspects, the above conditions play an important role in verifying whether $E_{J}$ can be determined exactly or not. For the class of symmetric DMCs, we can use the conditions $t R_{c r}^{(s)}(Q) \geq$ $R_{c r}(W)$ and $t R_{c r}^{(s)}(Q)<R_{c r}(W)$ to derive explicit formulas for $E_{J}$, see Example 2. In Section IV, we apply Theorem 2 to establish the conditions for which the JSCC exponent is larger than the tandem coding exponent. Note that when $t R_{c r}^{(s)}(Q) \leq$
$R_{c r}(W)$, the source-channel random-coding bound admits a simple expression

$$
\underline{E}_{r}(Q, W, t)=E_{0}(1, W)-t E_{s}(1, Q) .
$$

Consequently, we have the following statement.

Corollary 2: If $t R_{c r}^{(s)}(Q) \leq R_{c r}(W)$, then Csiszár's random-coding bound and Gallager's lower bound (27) are identical.

Example 2 (DMS and Symmetric DMC): Consider a DMS $\{Q: \mathcal{S}\}$ and a symmetric ${ }^{4}$ DMC $\{W: \mathcal{X} \rightarrow \mathcal{Y}\}$ with rate $t$, where the channel transition matrix $W$ can be partitioned along its columns into submatrices $W_{1}, W_{2}, \ldots, W_{s}$, such that in each $W_{i}$ with size $|\mathcal{X}| \times\left|\mathcal{Y}_{i}\right|$, each row is a permutation of each other row and each column is a permutation of each other column. Denote the transition probabilities in any column of submatrix $W_{i}$, $i=1,2, \ldots, s$, by $\left\{p_{i 1}, p_{i 2}, \ldots, p_{i|\mathcal{X}|}\right\}$. Then both $E_{0}(\rho, W)$ and the channel capacity are achieved by the uniform distribution $P_{X}=1 /|\mathcal{X}|$ and have the form

$E_{0}(\rho, W)=(1+\rho) \log |\mathcal{X}|-\log \left\{\sum_{i=1}^{s}\left|\mathcal{Y}_{i}\right|\left(\sum_{j=1}^{|\mathcal{X}|} p_{i j}^{\frac{1}{1+\rho}}\right)^{1+\rho}\right\}$

and

$$
C=\log |\mathcal{X}|-\frac{1}{|\mathcal{X}|} \sum_{i=1}^{s}\left|\mathcal{Y}_{i}\right|\left(\sum_{j=1}^{|\mathcal{X}|} p_{i j}\right) H\left(P_{i}^{(0)}\right)
$$

where the tilted distribution $P_{i}^{(\alpha)}, \alpha \geq 0$, for each $i=1,2, \ldots, s$, is defined on $I_{\mathcal{X}} \triangleq\{1,2, \ldots,|\mathcal{X}|\}$ by

$$
P_{i}^{(\alpha)}(j) \triangleq \frac{p_{i j}^{\frac{1}{1+\alpha}}}{\left(\sum_{j=1}^{|\mathcal{X}|} p_{i j}^{\frac{1}{1+\alpha}}\right)}, \quad j \in I_{\mathcal{X}} .
$$

Since now $E_{0}(\rho, W)$ is a concave and differentiable function of $\rho$, the bounds $\underline{E}_{r}(Q, W, t)$ and $\bar{E}_{s p}(Q, W, t)$ can be analytically obtained. If

$$
\frac{1}{|\mathcal{X}|} \sum_{i=1}^{s}\left|\mathcal{Y}_{i}\right|\left(\sum_{j=1}^{|\mathcal{X}|} p_{i j}\right) H\left(P_{i}^{(0)}\right)+t H(Q)<\log |\mathcal{X}|
$$

and

$$
\frac{\sum_{i=1}^{s}\left|\mathcal{Y}_{i}\right|\left(\sum_{j=1}^{|\mathcal{X}|} \sqrt{p_{i j}}\right)^{2} H\left(P_{i}^{(1)}\right)}{\sum_{i=1}^{s}\left|\mathcal{Y}_{i}\right|\left(\sum_{j=1}^{|\mathcal{X}|} \sqrt{p_{i j}}\right)^{2}}+t H\left(Q^{(1)}\right) \geq \log |\mathcal{X}|
$$

then the source-channel exponent is positive and is exactly determined by

$$
\begin{aligned}
& E_{J}(Q, W, t)=\left(1+\bar{\rho}^{*}\right) \log |\mathcal{X}| \\
& -\log \left\{\left[\sum_{i=1}^{s}\left|\mathcal{Y}_{i}\right|\left(\sum_{j=1}^{|\mathcal{X}|} p_{i j}^{\frac{1}{1+\bar{\rho}^{*}}}\right)^{1+\bar{\rho}^{*}}\right]\left(\sum_{s \in \mathcal{S}} Q^{\frac{1}{1+\bar{\rho}^{*}}}(s)\right)^{t\left(1+\bar{\rho}^{*}\right)}\right\}
\end{aligned}
$$

${ }^{4}$ Here, symmetry is defined in the Gallager sense [23, p. 94]; it is a generalization of the standard notion of symmetry [16] (which corresponds to $s=1$ above). 
where $\bar{\rho}^{*}$ is the unique root of the equation

$$
\frac{\sum_{i=1}^{s}\left|\mathcal{Y}_{i}\right|\left(\sum_{j=1}^{|\mathcal{X}|} p_{i j}^{\frac{1}{1+\rho}}\right)^{1+\rho} H\left(P_{i}^{(\rho)}\right)}{\sum_{i=1}^{s}\left|\mathcal{Y}_{i}\right|\left(\sum_{j=1}^{|\mathcal{X}|} p_{i j}^{\frac{1}{1+\rho}}\right)^{1+\rho}}+t H\left(Q^{(\rho)}\right)=\log |\mathcal{X}| .
$$

In the case when (37) does not hold, which means $t H(Q) \geq C$, $E_{J}(Q, W, t)=0$. When (37) holds but (38) does not hold, the right-hand side of (39) becomes the upper bound $\bar{E}_{s p}(Q, W, t)$ and meanwhile, $E_{J}$ is lower-bounded by $E_{0}(1, W)-t E_{s}(1, Q)$, where $E_{0}(\rho, W)$ is given by (36).

Now we apply the conditions (37) and (38) to a communication system with a binary source with distribution $\{q, 1-q\}$, a binary-symmetric channel (BSC) with crossover probability $\varepsilon$ and transmission rates $t=0.5,0.75,1$, and 1.25 . Note that

and

$$
R_{c r}(W)=1-h_{b}\left(\frac{\sqrt{\varepsilon}}{\sqrt{\varepsilon}+\sqrt{1-\varepsilon}}\right)
$$

$$
R_{c r}^{(s)}(Q)=h_{b}\left(\frac{\sqrt{q}}{\sqrt{q}+\sqrt{1-q}}\right)
$$

where $h_{b}(\cdot)$ is the binary entropy function. In Fig. 3, we partition the set of possible points for the $(\varepsilon, q)$ pairs into three regions: $\boldsymbol{A}, \boldsymbol{B}$, and $\boldsymbol{C}$. If $(\varepsilon, q) \in \boldsymbol{B}$, where conditions (37) and (38) hold, i.e., $t H(Q)<C$ and $t R_{c r}^{(s)}(Q) \geq R_{c r}(W)$, then the corresponding $E_{J}$ is positive and exactly known. ${ }^{5}$ Furthermore, if $(\varepsilon, q) \in \boldsymbol{C}$, then $E_{J}$ is bounded above (below, respectively) by the right-hand side of (39) $\left(E_{0}(1, W)-t E_{s}(1, Q)\right.$, respectively). When $(\varepsilon, q) \in \boldsymbol{A}$, where $t H(Q)>C, E_{J}$ is zero, and the error probability of this communication system converges to 1 for $n$ sufficiently large. So we are only interested in the cases when $(\varepsilon, q) \in \boldsymbol{B} \cup \boldsymbol{C}$.

Observation 4 (Csiszár's Expurgated Lower Bound): Using a similar approach (relying on Fenchel's Duality Theorem), we have also studied Csiszár's expurgated lower bound [18] to $E_{J}$ and obtained its equivalent expression in terms of Gallager's expurgated channel and source functions. We established the condition when the random-coding lower bound to $E_{J}$ can be improved by the expurgated bound. The reader may consult [57, Sec. 3.3] for a detailed discussion. Moreover, closed-form parametric expressions of the improved lower bound and its corresponding condition are provided in [57] for systems with equidistant DMCs.

${ }^{5}$ In light of the recent work in [11], where the random coding exponent $E_{r}(R, W)$ of the BSC is shown to be indeed the true value of the channel error exponent $E(R, W)$ for code rates $R$ in some interval directly below the channel critical rate (in other words, it is shown that for the BSC with its $\varepsilon$ above a certain threshold, $E_{r}(R, W)=E(R, W)$ for $R_{1} \leq R \leq C$ where $R_{1}$ can be less than $R_{c r}(W)$ [11]), we note via (1) and the lower bound in (22) and (23) that region $\boldsymbol{B}$ where $E_{J}$ is exactly known can be enlarged.
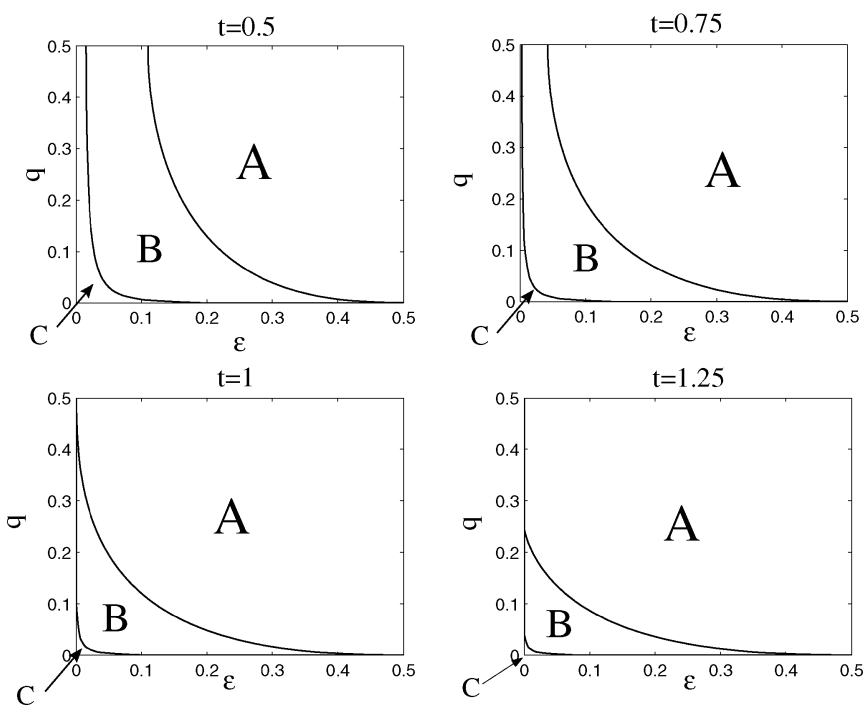

Fig. 3. The regions for the $(\varepsilon, q)$ pairs in the binary DMS $\{q, 1-q\}$ and BSC $(\varepsilon)$ system of Example 2 for different transmission rates $t$. Note that $E_{J}=0$ on the boundary between $\boldsymbol{A}$ and $\boldsymbol{B} ; E_{J}$ is exactly determined on the boundary between $\boldsymbol{B}$ and $\boldsymbol{C}$. In $\boldsymbol{A}, E_{J}=0$. In $\boldsymbol{B}, E_{J}$ is positive and known exactly. In $\boldsymbol{C}, E_{J}$ is positive and can be bounded above and below.

\section{WHEN IS JSCC WORTHWHILE: JSCC VERSUS TANDEM CODING EXPONENTS}

\section{A. Tandem Coding Error Exponent}

A tandem code $\left(f_{n}^{*}, \varphi_{n}^{*}\right) \triangleq\left(f_{c n} \circ f_{s n}, \varphi_{s n} \circ \varphi_{c n}\right)$ for a DMS $\{Q: \mathcal{S}\}$ and a DMC $\{W: \mathcal{X} \rightarrow \mathcal{Y}\}$ with block length $n$ and transmission rate $t$ (source symbols/channel use) is composed independently by a $(t n, M)$ block source code $\left(f_{s n}, \varphi_{s n}\right)$ defined by $f_{s n}: \mathcal{S}^{t n} \longrightarrow\{1,2, \ldots, M\}$ and $\varphi_{s n}:\{1,2, \ldots, M\} \longrightarrow \mathcal{S}^{t n}$ with source code rate

$$
R_{s} \triangleq \frac{\log M}{t n} \quad \text { source code bits/source symbol }
$$

and an $(n, M)$ block channel code $\left(f_{c n}, \varphi_{c n}\right)$ defined by $f_{c n}$ : $\{1,2, \ldots, M\} \longrightarrow \mathcal{X}^{n}$ and $\varphi_{c n}: \mathcal{Y}^{n} \longrightarrow\{1,2, \ldots, M\}$ with channel code rate

$$
R_{c} \triangleq \frac{\log M}{n} \quad \text { source code bits/channel use }
$$

where "o" means composition and $R_{s}$ and $R_{c}$ are independent of $n$. That is, blocks $s^{t n}$ of source symbols of length $t n$ are encoded as integers (indices) $f_{s n}\left(s^{t n}\right)$ from $\{1,2, \ldots, M\}$, and these integers are further encoded as blocks $x^{n}=f_{c n}\left[f_{s n}\left(s^{t n}\right)\right]$ of symbols from $\mathcal{X}$ of length $n$, transmitted, received as blocks $y^{n}$ of symbols from $\mathcal{Y}$ of length $n$. These received blocks $y^{n}$ are decoded as integers $\varphi_{c n}\left(y^{n}\right)$ from $\{1,2, \ldots, M\}$, and finally, these integers are decoded as blocks of source symbols $\varphi_{n}^{*}\left(y^{n}\right)=\varphi_{s n}\left[\varphi_{c n}\left(y^{n}\right)\right]$ of length $t n$. Thus, the probability of erroneously decoding the block is as shown in the equation at the bottom of the page, where $Q_{t n}$ and $P_{n, Y \mid X}$ are the $t n$ - and $n$-dimensional product distributions corresponding to $Q$ and $P_{Y \mid X}$. respectively.

$$
P_{e^{*}}^{(n)}(Q, W, t) \triangleq \sum_{\left\{\left(s^{t n}, y^{n}\right): \varphi_{s n}\left[\varphi_{c n}\left(y^{n}\right)\right] \neq s^{t n}\right\}} Q_{t n}\left(s^{t n}\right) P_{n, Y \mid X}\left(y^{n} \mid f_{c n}\left[f_{s n}\left(s^{t n}\right)\right]\right)
$$


Definition 2: The tandem coding error exponent $E_{T}(Q, W, t)$ is defined as the largest number $\widehat{E}$ for which there exists a sequence of tandem codes

$$
\left(f_{n}^{*}, \varphi_{n}^{*}\right)=\left(f_{c n} \circ f_{s n}, \varphi_{s n} \circ \varphi_{c n}\right)
$$

with transmission rate $t$ and block length $n$ such that

$$
\widehat{E} \leq \liminf _{n \rightarrow \infty}-\frac{1}{n} \log P_{e^{*}}^{(n)}(Q, W, t) .
$$

When there is no possibility of confusion, $E_{T}(Q, W, t)$ will often be written as $E_{T}$. In general, we know that $E_{J} \geq E_{T}$ since by definition tandem coding is a special case of JSCC. We are hence interested in determining the conditions for which $E_{J}>E_{T}$ for the same transmission rate $t$. Meanwhile, it immediately follows (from the JSCC theorem) that $E_{T}$ can be positive if and only if $t H(Q)<C$; otherwise, both $E_{J}$ and $E_{T}$ are zero.

By definition, the tandem coding exponent results from separately performing and concatenating optimal source and channel coding, which can be expressed by (e.g., see [17])

$$
\begin{aligned}
E_{T}(Q, W, t) & =\sup _{R_{s}, R_{c}: R_{c}=t R_{s}} \min \left\{t e\left(R_{s}, Q\right), E\left(R_{c}, W\right)\right\} \\
& =\sup _{R} \min \left\{t e\left(\frac{R}{t}, Q\right), E(R, W)\right\}
\end{aligned}
$$

where $e(R, Q)$ and $E(R, W)$ are the source and channel error exponents, respectively. Note that

$$
\sup _{R \leq t \log |\mathcal{S}|} t e\left(\frac{R}{t}, Q\right)=t e(\log |\mathcal{S}|, Q)=-t \log (|\mathcal{S}| \overline{Q(s)})
$$

where $\overline{Q(s)}$ is the geometric mean of the source probabilities, i.e.,

$$
\overline{Q(s)} \triangleq\left(\prod_{s \in \mathcal{S}} Q(s)\right)^{1 /|\mathcal{S}|} \leq 1 /|\mathcal{S}| .
$$

If $-t \log (|\mathcal{S}| \overline{Q(s)}) \geq E(t \log |\mathcal{S}|, W)$, then the graphs of $t e(R / t, Q)$ and $E(R, W)$ must have exactly one intersection $R_{o}$ and by (41)

$$
E_{T}(Q, W, t)=t e\left(\frac{R_{o}}{t}, Q\right)=E\left(R_{o}, W\right)
$$

since $t e(R / t, Q)$ is strictly increasing in $R \in[t H(Q), t \log |\mathcal{S}|]$ and $E(R, W)$ is nonincreasing in $R$. If

$$
-t \log (|\mathcal{S}| \overline{Q(s)})<E(t \log |\mathcal{S}|, W)
$$

then there is no intersection between $t e(R / t, Q)$ and $E(R, W)$. Recall (18) that $t e(R / t, Q)$ is infinite in the open interval $(t \log |\mathcal{S}|, \infty)$. In this case, we have that

$$
E_{T}(Q, W, t)=E(t \log |\mathcal{S}|, W)
$$

by (41). Without loss of generality, we get (44) at the bottom of the page, so that we can always write that $E_{T}(Q, W, t)=$ $E\left(R_{o}, W\right)$.
When the DMS is uniform, the optimal source coding operation reduces to the trivial enumerating (identity) function with $M=|S|^{t n}$ as the source is incompressible. Hence, only channel coding is performed in both JSCC and tandem coding and $E_{J}(Q, W, t)=E_{T}(Q, W, t)=E(t \log |\mathcal{S}|, W)$. Thus, our comparison of the two exponents is nontrivial only if the source is nonuniform and $t H(Q)<C$. Even though we know that $E_{J}$ is never worse than $E_{T}$, the following theorem gives a limit on how much $E_{J}$ can outperform $E_{T}$.

Theorem 3: JSCC exponent can at most be equal to double the tandem coding exponent, i.e.,

$$
E_{J}(Q, W, t) \leq 2 E_{T}(Q, W, t)
$$

with equality if $t R_{c r}^{(s)}(Q) \geq R_{c r}(W)$ and

$$
T_{s p}\left(\bar{\rho}^{*}, W\right)=t E_{s}\left(\bar{\rho}^{*}, Q\right)+2 t D\left(Q^{\left(\bar{\rho}^{*}\right)} \| Q\right) .
$$

Remark 4: Equivalently, this upper bound also implies that $E_{J}$ can at most exceed $E_{T}$ by $E_{J} / 2$, i.e.,

$$
E_{J}(Q, W, t)-E_{T}(Q, W, t) \leq \frac{1}{2} E_{J}(Q, W, t) .
$$

Proof: We first refer to the upper bound of $E_{J}(Q, W, t)$ given by Csiszár [17, Lemma 2] in (1)

$$
E_{J}(Q, W, t) \leq \min _{t H(Q) \leq R \leq t \log |\mathcal{S}|}\left[t e\left(\frac{R}{t}, Q\right)+E(R, W)\right]
$$

where $t e(R / t, W)$ is the source error exponent, which is strictly convex and increasing in $[t H(Q), t \log |\mathcal{S}|]$, and $E(R, W)$ is the channel error exponent, which is a positive and nonincreasing in $[0, C)$. Unlike the source exponent, the behavior of $E(R, W)$ is unknown for $R<R_{c r}(W)$. Let $C_{0}$ be the zero-error capacity of the channel $W$, i.e., $E(R, W)=\infty$ if and only if $R<C_{0}$ [23]. If $C_{0}>t \log |\mathcal{S}|$, obviously, we have

$$
E_{J}(Q, W, t)=E_{T}(Q, W, t)=+\infty .
$$

If $C_{0} \leq t \log |\mathcal{S}|$, the upper bound in (46) is finite and the minimum must be achieved by some rate, say $R_{m}$, in the interval $\left[C_{0}, t \log |\mathcal{S}|\right]$. Then

$$
\begin{aligned}
E_{J}(Q, W, t) & \stackrel{(\text { a) }}{\leq} t e\left(\frac{R_{m}}{t}, Q\right)+E\left(R_{m}, W\right) \\
& \stackrel{(\text { b) }}{\leq} t e\left(\frac{R_{o}}{t}, Q\right)+E\left(R_{o}, W\right) \\
& \stackrel{(\text { c) }}{\leq} 2 E\left(R_{o}, W\right) \\
& =2 E_{T}(Q, W, t) .
\end{aligned}
$$

Here, the equality in (a) holds if our computable upper and lower bounds, $\bar{E}_{s p}(Q, W, t)$ and $\underline{E}_{r}(Q, W, t)$, are equal. To ensure this, we need the condition $t R_{c r}^{(s)}(Q) \geq R_{c r}(W)$ by Theorem 2 . The equality in (b) holds if $R_{m}=R_{o}$ by definition of $R_{m}$. The equality (c) holds if and only if there

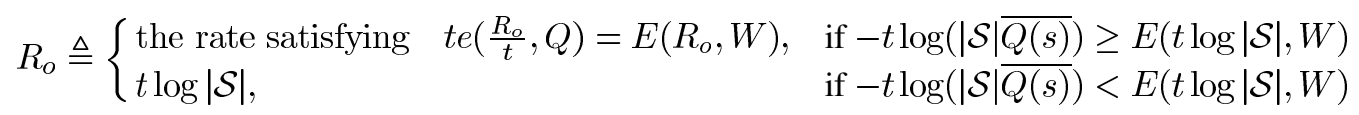


is an intersection between $t e(R / t, W)$ and $E(R, W)$, i.e., $t e\left(R_{o} / t, Q\right)=E\left(R_{o}, W\right)$. Now taking these considerations together, and applying Theorem 2 again, we conclude that $E_{J}=2 E_{T}$ if $t R_{c r}^{(s)}(Q) \geq R_{c r}(W)$ and

$T_{s p}\left(\bar{\rho}^{*}, W\right)-t E_{s}\left(\bar{\rho}^{*}, Q\right)=2 t e\left(\bar{R}_{m} / t, Q\right)=2 t D\left(Q^{\left(\bar{\rho}^{*}\right)} \| Q\right)$.

Observation 5: The condition for the equality states that, if the minimum in the expression of $\underline{E}_{r}(Q, W, t)$ given in (23) is attained at the intersection of $t e\left(\frac{R}{t}, W\right)$ and $E_{r}(R, W)$ which is no less than the critical rate of the channel, then the JSCC exponent is twice as large as the tandem coding exponent. In that case, the rate of decay of the error probability for the JSCC system is double that for the tandem coding system. In other words, for the same probability of error $P_{e}$, the delay of (optimal) JSCC is approximately half of the delay of (optimal) tandem coding

$$
P_{e} \approx 2^{-n E_{T}(Q, W, t)}=2^{-\frac{n}{2} E_{J}(Q, W, t)} \text { for } n \text { sufficiently large. }
$$

\section{B. Sufficient Conditions for Which $E_{J}>E_{T}$}

In the following, we will use our previous results to derive computable sufficient conditions for which $E_{J}>E_{T}$. We first define $\gamma$ in (47) at the bottom of the page, such that the source error exponent $t e(R / t, Q)$ has a parametric expression at $R_{c r}(W)$

$$
t e\left(\frac{R_{c r}(W)}{t}, Q\right)=t D\left(Q^{(\gamma)} \| Q\right) \text {. }
$$

Note that $\gamma$ is well defined only if $R_{c r}(W) \leq t \log |\mathcal{S}|$. Denote

$$
T\left(\bar{\rho}^{*}\right) \triangleq T_{s p}\left(\bar{\rho}^{*}, W\right)-t E_{s}\left(\bar{\rho}^{*}, Q\right) .
$$

Theorem 4: Let $R_{c r}(W) \leq t \log |\mathcal{S}|$. If

$$
\max \left\{t R_{c r}^{(s)}(Q), E_{o}(1, W)-t D\left(Q^{(\gamma)} \| Q\right)\right\} \geq R_{c r}(W)
$$

then

$$
E_{J}(Q, W, t)>E_{T}(Q, W, t)
$$

More precisely, we have the following bounds.

a) If

$$
\begin{aligned}
& \min \left\{t R_{c r}^{(s)}(Q), E_{o}(1, W)-t D\left(Q^{(\gamma)} \| Q\right)\right\} \geq R_{c r}( \\
& \text { then } \\
& E_{J}(Q, W, t)-E_{T}(Q, W, t) \\
& \quad \geq \frac{1}{2} T\left(\bar{\rho}^{*}\right)-\left|\frac{1}{2} T\left(\bar{\rho}^{*}\right)-t D\left(Q^{\left(\bar{\rho}^{*}\right)} \| Q\right)\right| \geq 0
\end{aligned}
$$

where the two equalities in (51) cannot hold simultaneously. b) If

$$
t R_{c r}^{(s)}(Q) \geq R_{c r}(W)>E_{o}(1, W)-t D\left(Q^{(\gamma)} \| Q\right)
$$

then

$$
\begin{aligned}
& E_{J}(Q, W, t)-E_{T}(Q, W, t) \\
& \quad>T\left(\bar{\rho}^{*}\right)-t D\left(Q^{(\gamma)} \| Q\right) \geq 0 .
\end{aligned}
$$

c) If

$$
E_{o}(1, W)-t D\left(Q^{(\gamma)} \| Q\right) \geq R_{c r}(W)>t R_{c r}^{(s)}(Q)
$$

then

$$
\begin{aligned}
E_{J}(Q, W, t)- & E_{T}(Q, W, t) \\
& \geq R_{c r}(W)-t E_{s}(1, Q)>0 .
\end{aligned}
$$

Proof: We shall show that, in each of the three cases, a), b), and c), we have $E_{J}>E_{T}$.

a). Assume $t R_{c r}^{(s)}(Q) \geq R_{c r}(W)$ and

$$
E_{o}(1, W)-t D\left(Q^{(\gamma)} \| Q\right) \geq R_{c r}(W) .
$$

By definition of $\gamma$, we have

$$
t D\left(Q^{(\gamma)} \| Q\right)=t e\left(R_{c r}(W) / t, Q\right),
$$

see (18) and (48). Thus, the latter condition is equivalent to $E\left(R_{c r}(W), W\right) \geq t e\left(R_{c r}(W) / t, Q\right)$ and by (12) and the related discussion it guarantees that $R_{o} \geq R_{c r}(W)$, where $R_{o}$ is defined in (44). According to Theorem 2, when $t R_{c r}^{(s)}(Q) \geq R_{c r}(W), \bar{E}_{s p}(Q, W, t)$ is attained by $\bar{R}_{m} \geq R_{c r}(W)$ and $E_{J}$ is determined by

$$
E_{J}(Q, W, t)=t e\left(\frac{\bar{R}_{m}}{t}, Q\right)+E_{s p}\left(\bar{R}_{m}, W\right) .
$$

Since $R_{o} \geq R_{c r}(W), E_{T}$ is determined by $E_{s p}\left(R_{o}, W\right)$. If $R_{o} \neq \bar{R}_{m}$, we must have

$$
E_{T}(Q, W, t)<\max \left\{t e\left(\frac{\bar{R}_{m}}{t}, Q\right), E_{s p}\left(\bar{R}_{m}, W\right)\right\}
$$

because $t e(R / t, Q)$ is strictly increasing and $E_{s p}(R, W)$ is strictly decreasing at $\bar{R}_{m}$. Thus,

$$
\begin{aligned}
& E_{J}(Q, W, t)-E_{T}(Q, W, t) \\
& \quad>\min \left\{t e\left(\frac{\bar{R}_{m}}{t}, Q\right), E_{r}\left(\bar{R}_{m}, W\right)\right\} \geq 0
\end{aligned}
$$

where equality holds if $\bar{R}_{m}=C$. If $R_{o}=\bar{R}_{m}$, then immediately

$$
\begin{aligned}
E_{J}(Q, W, t)-E_{T}(Q, W, t) & =t e\left(\frac{\bar{R}_{m}}{t}, Q\right) \\
& =t D\left(Q^{\left(\bar{\rho}^{*}\right)} \| Q\right)
\end{aligned}
$$

where the above is positive since $\bar{\rho}^{*}>0$ by Lemma 5 , part 1). Note also that in this case $t e\left(\bar{R}_{m} / t, Q\right)=$ $E_{r}\left(\bar{R}_{m}, W\right)$, so (54) and (55) can be summarized by (51).

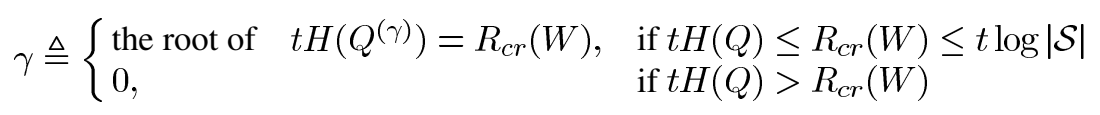


b). In this case, we have $\bar{R}_{m} \geq R_{c r}(W)>R_{o}$. We can upper-bound $E_{T}$ by

$$
\begin{array}{r}
E_{T}(Q, W, t)=t e\left(\frac{R_{o}}{t}, Q\right)<t e\left(\frac{R_{c r}(W)}{t}, Q\right) \\
=t D\left(Q^{(\gamma)} \| Q\right)
\end{array}
$$

and hence,

$$
\begin{aligned}
& E_{J}(Q, W, t)-E_{T}(Q, W, t) \\
& \quad>T_{s p}\left(\bar{\rho}^{*}, W\right)-t E_{s}\left(\bar{\rho}^{*}, Q\right)-t D\left(Q^{(\gamma)} \| Q\right) .
\end{aligned}
$$

The preceding lower bound must be nonnegative since

$$
\begin{aligned}
& T_{s p}\left(\bar{\rho}^{*}, W\right)-t E_{s}\left(\bar{\rho}^{*}, Q\right)-t D\left(Q^{(\gamma)} \| Q\right) \\
& \quad=E_{r}\left(\bar{R}_{m}, W\right)+t\left[e\left(\frac{\bar{R}_{m}}{t}, Q\right)-e\left(\frac{R_{c r}(W)}{t}, Q\right)\right] \\
& \geq E_{r}\left(\bar{R}_{m}, W\right) \\
& \geq 0
\end{aligned}
$$

and it is equal to 0 if $R_{c r}(W)=\bar{R}_{m}=C$.

c). In this case, we have $R_{o} \geq R_{c r}(W)>\underline{R}_{m}$ and from (35) $E_{J}$ is bounded by

$$
E_{J}(Q, W, t) \geq E_{0}(1, W)-t E_{s}(1, Q) .
$$

On the other hand, by the monotonicity of $E_{r}(R, W)$, we can upper-bound $E_{T}$ by

$$
\begin{aligned}
E_{T}(Q, W, t) & =E_{r}\left(R_{o}, W\right) \leq E_{r}\left(R_{c r}(W), W\right) \\
& =E_{0}(1, W)-R_{c r}(W) .
\end{aligned}
$$

Thus, we obtain

$$
E_{J}(Q, W, t)-E_{T}(Q, W, t) \geq R_{c r}(W)-t E_{s}(1, Q) .
$$

The above is positive since

$$
\begin{aligned}
E_{0}(1, W)-t E_{s}(1, Q) & =t e\left(\frac{\underline{R}_{m}}{t}, Q\right)+E_{r}\left(\underline{R}_{m}, W\right) \\
& >E_{r}\left(\underline{R}_{m}, W\right) \\
& >E_{r}\left(R_{c r}(W), W\right) \\
& =E_{0}(1, W)-R_{c r}(W)
\end{aligned}
$$

where the first inequality follows from the fact that $\underline{R}_{m}>$ $t H(Q)$ by Lemma 5 and Corollary 1.

As pointed out in the proof, the condition $t R_{c r}^{(s)}(Q) \geq$ $R_{c r}(W)$ means that the JSCC exponent $E_{J}$ is achieved at a rate no less than $R_{c r}(W)$. The second condition,

$$
E_{o}(1, W)-t D\left(Q^{(\gamma)} \| Q\right) \geq R_{c r}(W)
$$

means that the tandem coding exponent $E_{T}$ is achieved at a rate no less than $R_{c r}(W)$. Hence, (50) in Theorem 4 states that $E_{J}$ would be strictly larger than $E_{T}$ if either $E_{J}$ or $E_{T}$ is determined exactly. Conversely, if the conditions in Theorem 4 are not satisfied, then neither $E_{J}$ nor $E_{T}$ are exactly known. Nevertheless, if the lower bound of $E_{J}$ is strictly larger than the upper bound of $E_{T}$, then we must have $E_{J}>E_{T}$. Hence, we obtain the following sufficient conditions.

Theorem 5: Let $E_{e x}(0, W)<\infty$ and let $t \log |\mathcal{S}| \geq$ $R_{c r}(W)$, where $E_{e x}(R, W)$ is the expurgated channel error exponent [23]. If

$$
\begin{aligned}
E_{0}(1, W) & -t E_{s}(1, Q) \\
\geq E_{R_{l}} & \triangleq \frac{k_{1} k_{2} t \log |\mathcal{S}|+k_{2} t \log (|\mathcal{S}| \overline{Q(s)})+k_{1} E_{e x}(0, W)}{k_{1}-k_{2}}
\end{aligned}
$$
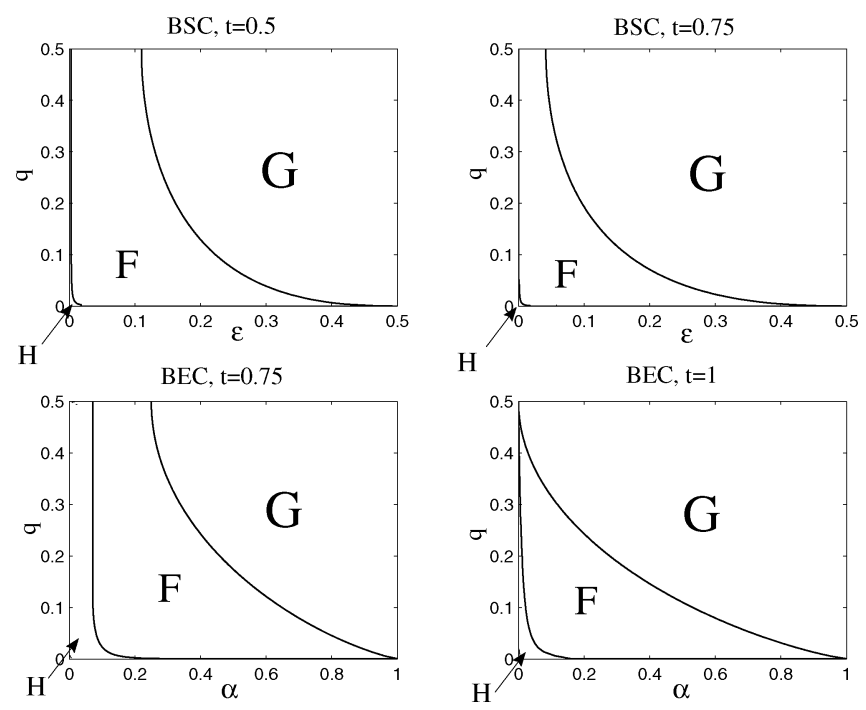

Fig. 4. The regions for binary DMS-BSC $(q, \varepsilon)$ pairs and binary DMS-BEC $(q, \alpha)$ pairs under different transmission rates $t$. In region $\boldsymbol{F}$ (including the boundary between $\boldsymbol{F}$ and $\boldsymbol{H}$ ), $E_{J}>E_{T}>0$; in region $\boldsymbol{G}$ (including the boundary between $\boldsymbol{G}$ and $\boldsymbol{F}), E_{J}=E_{T}=0$; and in region $\boldsymbol{H}, E_{J} \geq E_{T}>0$.

where

$$
\begin{aligned}
k_{1} & =\frac{D\left(Q^{(1)} \| Q\right)+\log (|\mathcal{S}| \overline{Q(s)})}{H\left(Q^{(1)}\right)-\log |\mathcal{S}|} \\
\text { and } \quad k_{2} & =\frac{E_{0}(1, W)-E_{e x}(0, W)}{R_{c r}(W)}-1
\end{aligned}
$$

then $E_{J}(Q, W, t)>E_{T}(Q, W, t)$.

Theorem 6: Let $t \log |\mathcal{S}| \geq R_{c r}(W)$. If

$$
E_{0}(1, W)-t E_{s}(1, Q) \geq t D\left(Q^{(\gamma)} \| Q\right)
$$

where $\gamma$ is defined in (47), then $E_{J}(Q, W, t)>E_{T}(Q, W, t)$.

In Theorems 5 and 6, we establish the sufficient conditions by comparing the source-channel random-coding bound derived in Theorem 2, with the upper bound of tandem coding exponent obtained by using the geometric characteristics of $e(R, W)$ and $E(R, W)$. For the proofs of Theorems 5 and 6 , the reader may refer to [57, Appendices B and C]. These conditions can be readily computed since it only requires the knowledge of $R_{c r}(W)$ and $E_{e x}(0, W)$. Note that the condition $E_{e x}(0, W)<$ $\infty$ in Theorem 5 is satisfied by the DMCs with zero-error capacity equal to 0 , see $[19$, p. 187]. Thus, Theorem 5 applies to equidistant channels, in particular, to every channel with binary input alphabet. An expression of $E_{e x}(0, W)$ for the DMC with 0 zero-error capacity is given in [23, Problem 5.24].

Example 3 (When Does the JSCC Exponent Outperform the Tandem Coding Exponent?): We apply Theorems 4-6 to the binary DMS with distribution $\{q, 1-q\}$ and BSC with crossover probability $\varepsilon$, and the binary DMS $\{q, 1-q\}$ and the binary erasure channel (BEC) with erasure probability $\alpha$, under different transmission rates $t$. If any one of the conditions in these theorems holds, then $E_{J}>E_{T}$. The above conditions are summarized by Region $\boldsymbol{F}$ in Fig. 4. Indeed, Region $\boldsymbol{F}$ shows that $E_{J}>E_{T}$ for a wide range of $(\varepsilon, q)$ or $(\alpha, q)$ pairs. Region $\boldsymbol{G}$ consists of the pairs $(\varepsilon, q)$ or $(\alpha, q)$ such that $t H(Q) \geq C$; in this case, $E_{J}=E_{T}=0$. Finally, when $(\varepsilon, q)$ or $(\alpha, q)$ falls 
TABLE I

$E_{J} / E_{T}$ FOR the Binary DMS and BSC Pairs of Example 4. "N/A" Means That $t H(Q)>C$ Such That $E_{J}=E_{T}=0$. “ $\nmid$ ” Means That This QUANTITY IS ONLY A LOWER BOUND FOR $E_{J} / E_{T}$

\begin{tabular}{||c|c|c|c|c||}
\hline \hline$E_{J} / E_{T}$ & $\mathrm{t}=0.5, \mathrm{q}=0.1$ & $\mathrm{t}=0.75, \mathrm{q}=0.1$ & $\mathrm{t}=0.75, \mathrm{q}=0.15$ & $\mathrm{t}=1, \mathrm{q}=0.05$ \\
\hline$\varepsilon=0.0005$ & $1.0^{\dagger}$ & $1.60^{\dagger}$ & $1.55^{\dagger}$ & $1.86^{\dagger}$ \\
\hline$\varepsilon=0.001$ & $1.0^{\dagger}$ & $1.70^{\dagger}$ & $1.68^{\dagger}$ & $1.93^{\dagger}$ \\
\hline$\varepsilon=0.005$ & $1.35^{\dagger}$ & $1.94^{\dagger}$ & 1.89 & 1.99 \\
\hline$\varepsilon=0.01$ & $1.70^{\dagger}$ & 1.95 & 1.91 & 2.0 \\
\hline$\varepsilon=0.04$ & 1.85 & 1.97 & 1.95 & 2.0 \\
\hline$\varepsilon=0.08$ & 1.91 & 1.99 & 1.96 & 2.0 \\
\hline$\varepsilon=0.12$ & 1.95 & 1.97 & 2.0 & 2.0 \\
\hline$\varepsilon=0.16$ & 1.96 & 1.95 & N/A & 2.0 \\
\hline$\varepsilon=0.2$ & 1.86 & N/A & N/A & N/A \\
\hline \hline
\end{tabular}

DMC

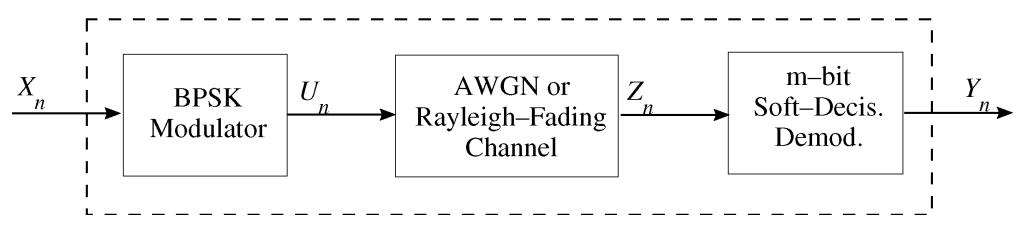

Fig. 5. Binary-input AWGN or Rayleigh-fading channel with finite output quantization.

in Region $\boldsymbol{H}$, we are not sure whether $E_{J}$ is still strictly larger than $E_{T}$.

Example 4 (By How Much can the JSCC Exponent be Larger Than the Tandem Coding Exponent?): In the last example, we have seen that $E_{J}>E_{T}$ holds for a wide large class of source-channel pairs. Now we evaluate the performance of $E_{J}$ over $E_{T}$ by looking at the ratio of the two quantities. Recall that when Theorem 4 part a) is satisfied, both $E_{J}$ and $E_{T}$ are exactly determined. In this case, we can directly compute $E_{J}$ (using the results of Section III) and $E_{T}$ (using (42) and (43)). When $E_{J}$ (respectively, $E_{T}$ ) is not known, i.e., when $t R_{c r}^{(s)}(Q)<R_{c r}(W)$ (respectively, $\left.E_{o}(1, W)-t D\left(Q^{(\gamma)} \| Q\right)<R_{c r}(W)\right)$, we can calculate the lower bound ${ }^{6}$ of $E_{J}$ (respectively, the upper bound of $E_{T}$ ) instead and thus obtain a lower bound for $E_{J} / E_{T}$. Specifically, when

$$
E_{o}(1, W)-t D\left(Q^{(\gamma)} \| Q\right)<R_{c r}(W) \leq R_{c r}^{(s)}(Q)
$$

or equivalently when $R_{o}<R_{c r}(W) \leq \bar{R}_{m}$, we can bound $E_{T}$ by

$$
E_{T}(Q, W, t) \leq \min \left\{t D\left(Q^{(\gamma)} \| Q\right), E_{s p}\left(R_{s}, W\right)\right\}
$$

where $R_{s}$ is the intersection of $E_{s p}(R, W)$ and $t e(R / t, Q)$ if any; otherwise, $R_{s}=t \log |S|$. When

$$
E_{o}(1, W)-t D\left(Q^{(\gamma)} \| Q\right)<R_{c r}(W)
$$

and $R_{c r}^{(s)}(Q)<R_{c r}(W)$, we bound $E_{T}$ by

$$
E_{T}(Q, W, t) \leq E_{s p}\left(R_{s}, W\right) .
$$

${ }^{6}$ Here we only use the random-coding lower bound of $E_{J}$. In [57, Example 5], we also calculated the expurgated lower bound of $E_{J}$, and chose the larger one in the computation of the lower bound for $E_{J} / E_{T}$. In this case, a slight improvement is noticed for some low values of $\varepsilon$ (see [57, Table I]).
Table I exhibits $E_{J} / E_{T}$ (or its lower bound, which must be no less than 1) for the binary DMS $\{q, 1-q\}$ and BSC $(\varepsilon)$ system under transmission rates $t=0.5,0.75$, and 1 . It is seen that the ratio $E_{J} / E_{T}$ can be very close to 2 (its upper bound) for many $(q, \varepsilon)$ pairs. For other systems, we have similar results: $E_{J}$ substantially outperforms $E_{T}$. For instance, for binary DMS $\{q, 1-q\}$ and $\operatorname{BEC}(\alpha)$ with $t=1$, we note that $E_{J} / E_{T} \geq$ 1.4 for a wide range of $(q, \alpha)^{\prime}$ 's; for ternary DMS and BSC or for DMS and ternary symmetric channel, if transmission rate $t$ is chosen suitably (such that $t H(Q)<C$ ), we obtain that $E_{J} / E_{T} \geq 1.5$ for many source-channel pairs.

\section{Power Gain Due to JSCC for DMS Over Binary-Input AWGN and Rayleigh-Fading Channels With Finite Output Quantization}

It is well known that $M$-ary modulated additive white Gaussian noise (AWGN) and memoryless Rayleigh-fading channels can be converted to a DMC when finite quantization is applied at their output. For example, as illustrated in [4], [41], we know that the concatenation of a binary phase-shift keying (BPSK) modulated AWGN or Rayleigh-fading channel with $m$-bit soft-decision demodulation is equivalent to a binary-input, $2^{m}$-output DMC (cf. Fig. 5). We next study the JSCC and tandem coding exponent for a system involving such channels to assess the potential benefits of JSCC over tandem coding in terms of power or channel signal-to-noise ratio (SNR) gains.

We assume that the BPSK signal $U_{n} \in\{-1,+1\}$ corresponding to the signal input $X_{n}$ is of unit energy, and $V_{n}$ is a zero-mean independent and identically distributed (i.i.d.) Gaussian random process with variance $N_{o} / 2$. The channel $\mathrm{SNR}$ is defined by $\mathrm{SNR} \triangleq E\left[U_{n}^{2}\right] / E\left[V_{n}^{2}\right]=2 / N_{o}$ and the received signal is

$$
Z_{n}=A_{n} U_{n}+V_{n}, \quad n=1,2, \ldots
$$


where $A_{n}$ is 1 for the AWGN channel (no fading), and for the Rayleigh-fading channel, $\left\{A_{n}\right\}$ is the amplitude fading process assumed to be i.i.d. with probability density function (pdf)

$$
f_{A}(a)= \begin{cases}2 a e^{-a^{2}}, & \text { if } a>0 \\ 0 & \text { otherwise }\end{cases}
$$

such that $E\left[A_{n}^{2}\right]=1$. We also assume for the Rayleigh-fading channel that $A_{n}, U_{n}$, and $V_{n}$ are independent of each other, and the values of $A_{n}$ are not available at the receiver. At the receiver, as shown in Fig. 5, each $Z_{n} \in \mathbb{R}$ is demodulated via an $m$-bit uniform scalar quantizer with quantization step $\Delta$ to yield $Y_{n} \in$ $\{0,1\}^{m}$. If the channel input alphabet is $\mathcal{X}=\{0,1\}$ and the channel output alphabet is $\mathcal{Y}=\left\{0,1,2, \ldots, 2^{m}-1\right\}$, then the transition probability matrix $\Pi$ is given by

$$
\Pi=\left[\pi_{i j}\right], \quad i \in \mathcal{X}, j \in \mathcal{Y}
$$

where

$$
\begin{aligned}
& \pi_{i j} \triangleq P(Y=j \mid X=i)=\mathcal{Q}(\left.\left(T_{j-1}-(2 i-1)\right) \sqrt{\mathrm{SNR}}\right) \\
&-\mathcal{Q}\left(\left(T_{j}-(2 i-1)\right) \sqrt{\mathrm{SNR}}\right)
\end{aligned}
$$

for the AWGN channel [41], and

$$
\pi_{i j} \triangleq P(Y=j \mid X=i)=F_{Z \mid X}\left(T_{j} \mid i\right)-F_{Z \mid X}\left(T_{j-1} \mid i\right)
$$

for the Rayleigh-fading channel [4]. Here

$$
F_{Z \mid X}(z \mid i)=\operatorname{Pr}\{Z \leq z \mid Z=i\}
$$

is given by [4], [49]

$$
\begin{aligned}
F_{Z \mid X}(z \mid 1)= & 1-F_{Z \mid X}(-z \mid 0)=1-\mathcal{Q}\left(\frac{z}{\sqrt{N_{o} / 2}}\right) \\
& -\frac{e^{-\left(z^{2} /\left(N_{o}+1\right)\right)}}{\sqrt{N_{o}+1}} \times\left[1-\mathcal{Q}\left(\frac{z}{\sqrt{N_{o}\left(N_{o}+1\right) / 2}}\right)\right]
\end{aligned}
$$

where $\mathcal{Q}(x)$ is the complementary error function

$$
\mathcal{Q}(x)=\frac{1}{\sqrt{2 \pi}} \int_{x}^{\infty} \exp \left\{-t^{2} / 2\right\} d t
$$

and $\left\{T_{j}\right\}$ are the thresholds of the receiver's soft-decision quantizer given by

$$
T_{j}= \begin{cases}-\infty, & \text { if } j=-1 \\ \left(j+1-2^{m-1}\right) \Delta, & \text { if } j=0,1, \ldots, 2^{m}-2 \\ +\infty, & \text { if } j=2^{m}-1\end{cases}
$$

with uniform step-size $\Delta$. For each channel SNR, the suitable quantization step $\Delta$ is chosen as in [41], [4] to yield the maximum capacity of the binary-input $2^{m}$-output DMC.

We compute the JSCC and tandem coding exponents for the binary source and the binary-input $2^{m}$-output DMC converted from the AWGN (Rayleigh-fading, respectively) channel under transmission rate $t=0.75$ ( $t=1$, respectively), and illustrate the power gain due to JSCC. In Figs. 6 and 7, we plot $E_{J}$ and $E_{T}$ for binary DMS $Q=\{0.1,0.9\}$ and $m=1,2,3$ by varying the channel SNR (in decibels). We point out that in both figures, when SNR $\leq 6 \mathrm{~dB}$ for $m=2,3$ and when SNR $\leq 8 \mathrm{~dB}$ for $m=1, E_{J}$ and $E_{T}$ are determined exactly. We observe that for the same SNR, $E_{J}$ is almost twice as large as $E_{T}$. Further-

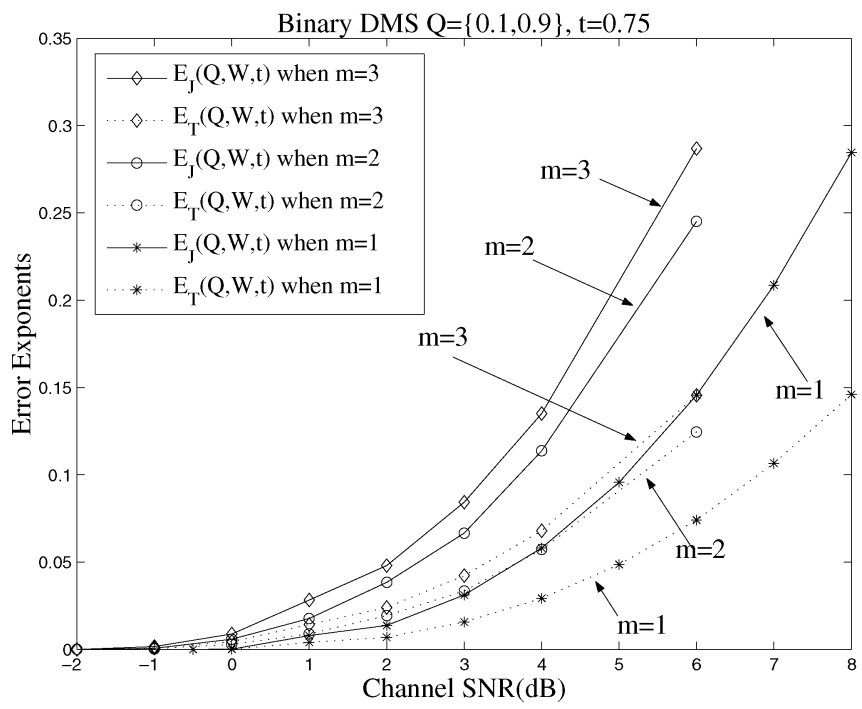

Fig. 6. The power gain due to JSCC for binary DMS and binary-input $2^{m}$-output DMC (AWGN channel) with $t=0.75$.

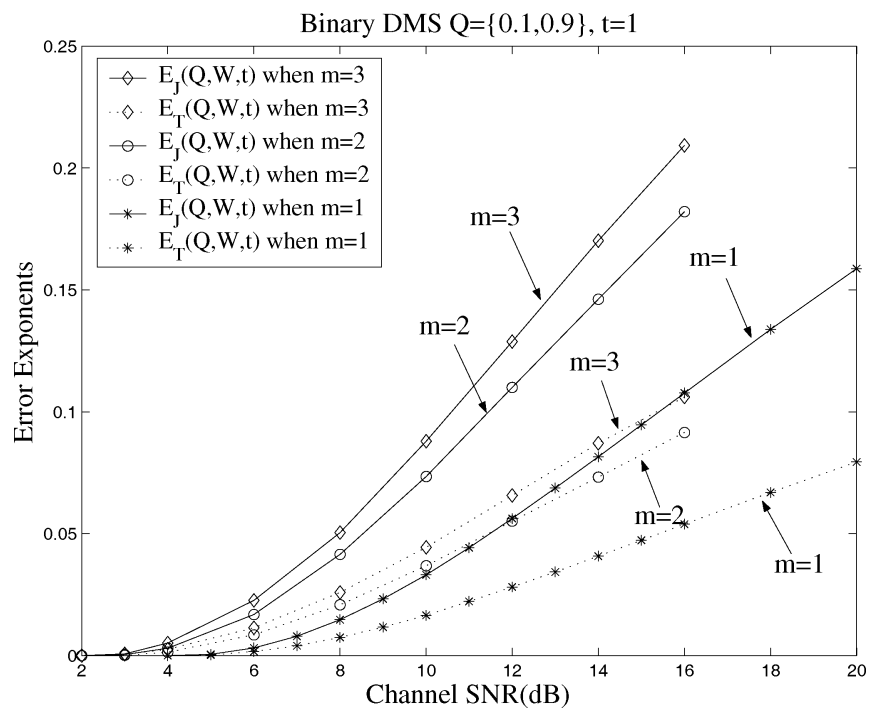

Fig. 7. The power gain due to JSCC for binary DMS and binary-input $2^{m}$-output DMC (Rayleigh-fading channel) with $t=1$.

more, for the same exponent and the same (asymptotic) encoding length, JSCC would yield the same probability of error as tandem coding with a power gain of more than $2 \mathrm{~dB}$. Similar behavior was noted for other values of transmission rate $t$.

\section{JSCC ERROR EXPONENT WITH HAMMING DISTORTION MEASURE}

Let $\mathcal{S}$ be a finite set and $d(\cdot, \cdot)$ be a distortion measure, i.e., a nonnegative valued function $d$ defined on $\mathcal{S} \times \mathcal{S}$ and extended to $\mathcal{S}^{n} \times \mathcal{S}^{n}$ by setting

$$
d\left(s^{n}, \widetilde{s}^{n}\right) \triangleq \frac{1}{n} \sum_{i=1}^{n} d\left(s_{i}, \widetilde{s}_{i}\right) .
$$

A JSC code with block length $n$ and transmission rate $t>0$ for a $t n$-length DMS $\{Q: \mathcal{S}\}$ and a DMC $\{W: \mathcal{X} \rightarrow \mathcal{Y}\}$ with a threshold $\Delta$ of tolerated distortion is a pair of mappings 
$f_{n}: \mathcal{S}^{t n} \longrightarrow \mathcal{X}^{n}$ and $\varphi_{n}: \mathcal{Y}^{n} \longrightarrow \mathcal{S}^{t n}$. The probability of the code exceeding the threshold $\Delta$ is given by

$$
\begin{aligned}
& P_{\Delta}^{(n)}(Q, W, t) \\
& \quad \triangleq \sum_{\left\{\left(s^{t n}, y^{n}\right): d\left(s^{t n}, \varphi_{n}\left(y^{n}\right)\right)>\Delta\right\}} Q_{t n}\left(s^{t n}\right) P_{n, Y \mid X}\left(y^{n} \mid f_{n}\left(s^{t n}\right)\right)
\end{aligned}
$$

where $Q_{t n}$ and $P_{n, Y \mid X}$ are the $t n$ - and $n$-dimensional product distributions corresponding to $Q$ and $P_{Y \mid X}$, respectively. $P_{\Delta}^{(n)}(Q, W, t)$ is also called the probability of excess distortion. We remark that for the JSCC with a distortion threshold, we allow that the source has a uniform distribution.

Definition 3: The JSCC error exponent $E_{J}^{\Delta}(Q, W, t)$ is defined as the largest number $E^{\Delta}$ for which there exists a sequence of JSC codes $\left(f_{n}, \varphi_{n}\right)$ with block length $n$ and transmission rate $t$ such that

$$
E^{\Delta} \leq \liminf _{n \rightarrow \infty}-\frac{1}{n} \log P_{\Delta}^{(n)}(Q, W, t)
$$

When there is no possibility of confusion, $E_{J}^{\Delta}(Q, W, t)$ will often be written $E_{J}^{\Delta}$. In [18], Csiszár proved that for a DMS $Q$ and a DMC $W$, the JSCC error exponent under distortion threshold $\Delta$ satisfies

$$
\underline{E}_{r}^{\Delta}(Q, W, t) \leq E_{J}^{\Delta}(Q, W, t) \leq \bar{E}_{s p}^{\Delta}(Q, W, t)
$$

where

$$
\underline{E}_{r}^{\Delta}(Q, W, t) \triangleq \inf _{R>0}\left[t F\left(\frac{R}{t}, Q, \Delta\right)+E_{r}(R, W)\right]
$$

and

$$
\bar{E}_{s p}^{\Delta}(Q, W, t) \triangleq \inf _{R>0}\left[t F\left(\frac{R}{t}, Q, \Delta\right)+E_{s p}(R, W)\right] .
$$

In the above

$$
F(R, Q, \Delta)=\inf _{P: R(P, \Delta)>R} D(P \| Q)
$$

is the source error exponent with a fidelity criterion [37] and $R(P, \Delta)$ is the rate distortion function (e.g., [16], [19]). $E_{r}(R, W)$ and $E_{s p}(R, W)$ are the random-coding and spherepacking bounds to the channel error exponent. Likewise, if the infimum in (58) or (59) is attained for a rate larger than the channel critical rate, then the lower and upper bounds coincide, and we can determine $E_{J}^{\Delta}$ exactly. Of course, the two bounds are nontrivial if and only if $t R(Q, \Delta)<C$ by the JSCC theorem.

It can be shown that $F(R, Q, \Delta)$ is a nondecreasing function in $R$. However, unlike $e(R, Q), F(R, Q, \Delta)$ is not necessarily convex or even continuous in $R$ [1], [37]. Therefore, it is hard to analytically compute the JSCC exponent $E_{J}^{\Delta}$ in general. In this section, we only address the computation of $E_{\vec{J}}^{\Delta}$ for a binary DMS and an arbitrary DMC under the Hamming distortion measure $d_{H}(\cdot, \cdot)$, given by

$$
d_{H}(s, \widetilde{s})= \begin{cases}1, & \text { if } s \neq \widetilde{s} \\ 0, & \text { if } s=\widetilde{s}\end{cases}
$$

We first need to derive a parametric form of $F(R, Q, \Delta)$. Define

$$
E_{s}^{\Delta}(\rho, Q) \triangleq(1+\rho) \log \left(q^{\frac{1}{1+\rho}}+(1-q)^{\frac{1}{1+\rho}}\right)-\rho h_{b}(\Delta) .
$$

Lemma 6: For binary DMS $Q \triangleq\{q, 1-q\}(q \leq 1 / 2)$ under the Hamming distortion measure (61) and distortion threshold $\Delta$ such that $\Delta \leq 1 / 2$, the following hold:

$$
\begin{aligned}
& F(R, Q, \Delta) \\
& = \begin{cases}+\infty, & R>1-h_{b}(\Delta) \\
\sup _{\rho \geq \rho_{0}}\left[\rho R-E_{s}^{\Delta}(\rho, Q)\right], & R(Q, \Delta)<R \leq 1-h_{b}(\Delta) \\
0, & R \leq R(Q, \Delta)\end{cases}
\end{aligned}
$$

where the rate-distortion function $R(Q, \Delta)=h_{b}(q)-h_{b}(\Delta)$ and $\rho_{0}=0$ if $q \geq \Delta$; otherwise, $R(Q, \Delta)=0$ and $\rho_{0}$ is the unique root of equation $H\left(Q^{(\rho)}\right)=h_{b}(\Delta)$ such that $\rho_{0}>0$.

The proof of this lemma is given in Appendix A. It can be easily verified that $F(R, Q, \Delta)$ is continuous and convex in $R \in\left(-\infty, 1-h_{b}(\Delta)\right]$ if $q \geq \Delta$ and $F(R, Q, \Delta)$ is continuous and convex in $R \in\left(0,1-h_{b}(\Delta)\right]$ and has a jump at $R=R(Q, \Delta)=0$ if $q<\Delta$. According to Lemma 6 , the source error exponent $t F(R / t, Q, \Delta)$ is the convex transform of $t E_{s}^{\Delta}(\rho, Q)$ in $\left[\rho_{0},+\infty\right)$. Define the binary divergence by

$$
\widetilde{D}(\Delta \| q) \triangleq \Delta \log \frac{\Delta}{q}+(1-\Delta) \log \frac{1-\Delta}{1-q} .
$$

Adopting the approach of Section III, we can apply Fenchel's Duality Theorem to $\underline{E}_{r}^{\Delta}(Q, W, t)$ and $\bar{E}_{s p}^{\Delta}(Q, W, t)$ and obtain equivalent computable bounds.

Theorem 7: Given a binary DMS $(q \leq 1 / 2)$ and a DMC $W$ under the Hamming distortion measure and distortion threshold $\Delta(\Delta \leq 1 / 2)$, the JSCC exponent satisfies the following.

1) Lower Bound: If $0 \leq \Delta<\sqrt{q} /(\sqrt{q}+\sqrt{1-q})$, then $\rho_{0}<1$ and

$$
\underline{E}_{r}^{\Delta}(Q, W, t)=\max _{\rho_{0} \leq \rho \leq 1}\left[T_{r}(\rho, W)-t E_{s}^{\Delta}(\rho, Q)\right] .
$$

Otherwise, if $\Delta \geq \sqrt{q} /(\sqrt{q}+\sqrt{1-q})$, then

$$
\underline{E}_{r}^{\Delta}(Q, W, t)=t \widetilde{D}(\Delta \| q)+E_{0}(1, W) .
$$

2) Upper Bound:

$$
\bar{E}_{s p}^{\Delta}(Q, W, t)=\sup _{\rho \geq \rho_{0}}\left[T_{s p}(\rho, W)-t E_{s}^{\Delta}(\rho, Q)\right] .
$$

Since the above result is a simple extension of the results in Section III, the proof is omitted and we hereby only provide the following remarks.

a) Similar to the lossless case, if $t\left(h_{b}(q)-h_{b}(\Delta)\right) \geq C$, then $\underline{E}_{r}^{\Delta}(Q, W, t)=\bar{E}_{s p}^{\Delta}(Q, W, t)=0$. If $R_{\infty}(W)>$ $t\left(1-h_{b}(\Delta)\right)$, then $\bar{E}_{s p}^{\Delta}(Q, W, t)=+\infty$. 
b) Note that when $\Delta \geq \sqrt{q} /(\sqrt{q}+\sqrt{1-q}), \underline{E}_{r}^{\Delta}(Q, W, t)$ in (58) is achieved at $R \downarrow 0^{+}$, and

$$
\begin{aligned}
& \underline{E}_{r}^{\Delta}(Q, W, t) \\
& \quad=\lim _{R \downarrow 0^{+}}\left[t F\left(\frac{R}{t}, Q, \Delta\right)+E_{r}(R, W)\right] \\
& \quad=\lim _{R \downarrow 0^{+}}\left[t \inf _{P: R(P, \Delta)>\frac{R}{t}} D(P \| Q)+E_{0}(1, W)-R\right] \\
& \quad=t \widetilde{D}(\Delta \| q)+E_{0}(1, W) .
\end{aligned}
$$

c) In the special case where the binary source is uniform, i.e., $q=1 / 2$, Theorem 7 reduces to

$$
\begin{aligned}
& \max _{0 \leq \rho \leq 1}\left[-\rho t\left(1-h_{b}(\Delta)\right)+T_{r}(\rho, W)\right] \\
& \quad \leq E_{J}^{\Delta}(Q, W, t) \leq \sup _{\rho \geq 0}\left[-\rho t\left(1-h_{b}(\Delta)\right)+T_{s p}(\rho, W)\right] .
\end{aligned}
$$

This is clearly equivalent to

$$
\begin{aligned}
E_{r}\left(t\left(1-h_{b}(\Delta)\right), W\right) & \leq E_{J}^{\Delta}(Q, W, t) \\
& \leq E_{s p}\left(t\left(1-h_{b}(\Delta)\right), W\right)
\end{aligned}
$$

by the definition of $T_{r}(\rho, W)$ and $T_{s p}(\rho, W)$. In other words, $E_{J}^{\Delta}$ is bounded by the channel random-coding and sphere-packing bounds at rate $t\left(1-h_{b}(\Delta)\right)$. If $t\left(1-h_{b}(\Delta)\right) \geq R_{c r}(W)$, then $E_{J}^{\Delta}$ is exactly determined.

d) When the source is nonuniform, $E_{s}^{\Delta}(\rho, Q)=E_{s}(\rho, Q)-$ $\rho h_{b}(\Delta)$ is strictly concave in $\rho$. In this case, the maximizer

$$
\bar{\rho}^{\Delta} \triangleq \arg \sup _{\rho \geq \rho_{0}}\left[T_{s p}(\rho, W)-t E_{s}^{\Delta}(\rho, Q)\right]
$$

is strictly larger than $\rho_{0}$ if $t\left(h_{b}(q)-h_{b}(\Delta)\right)<C$ and $R_{\infty}(W) \leq t\left(1-h_{b}(\Delta)\right)$. Particularly, $\bar{\rho}^{\Delta}<\infty$ if $R_{\infty}(W)<t\left(1-h_{b}(\Delta)\right)$. As counterparts of Lemma 5 and Corollary 1, it can be shown that the upper bound $\bar{E}_{s p}^{\Delta}(Q, W, t)$ in (59) is attained at $\bar{R}_{m}^{\Delta}=H\left(Q^{\left(\bar{\rho}^{\Delta}\right)}\right)-$ $h_{b}(\Delta)$ and the lower bound in (58) is attained at $\underline{R}_{m}^{\Delta}=H\left(Q^{\left(\rho^{\Delta}\right)}\right)-h_{b}(\Delta)$, where $\underline{\rho}^{\Delta}=\min \left\{\bar{\rho}^{\Delta}, 1\right\}$. Consequently, other similar results to the lossless case regarding these optimizers can be obtained.

Example 5: For a binary DMS $\{q, 1-q\}(q \leq 0.5)$ and a BSC $(\varepsilon)$ under transmission rate $t=1$, we compute the JSCC error exponent under the Hamming distortion measure with distortion threshold $\Delta\left(\Delta<\frac{1}{2}\right)$. In Fig. 8, if the pair $(\varepsilon, q)$ is located in region $B$, then the corresponding JSCC exponent can be determined exactly (the lower and upper bounds are equal). If $(\varepsilon, q)$ is located in region $\boldsymbol{C}_{1}$, then $E_{J}^{\Delta}$ is bounded by (65) and (67). If $(\varepsilon, q)$ is located in region $\boldsymbol{C}_{2}$, then $E_{J}^{\Delta}$ is bounded by (66) and (67). When $(\varepsilon, q) \in A, E_{J}^{\Delta}$ is zero, and the error probability of this communication system converges to 1 for $n$ sufficiently large. So we are only interested in the cases when $(\varepsilon, q) \in \boldsymbol{B} \cup \boldsymbol{C}_{1} \cup \boldsymbol{C}_{2}$.

Fig. 9 shows the JSCC error exponent lower bound of the binary DMS $\{q, 1-q\}(q \leq 0.5)$ and $\operatorname{BSC}(\varepsilon)$ pairs under
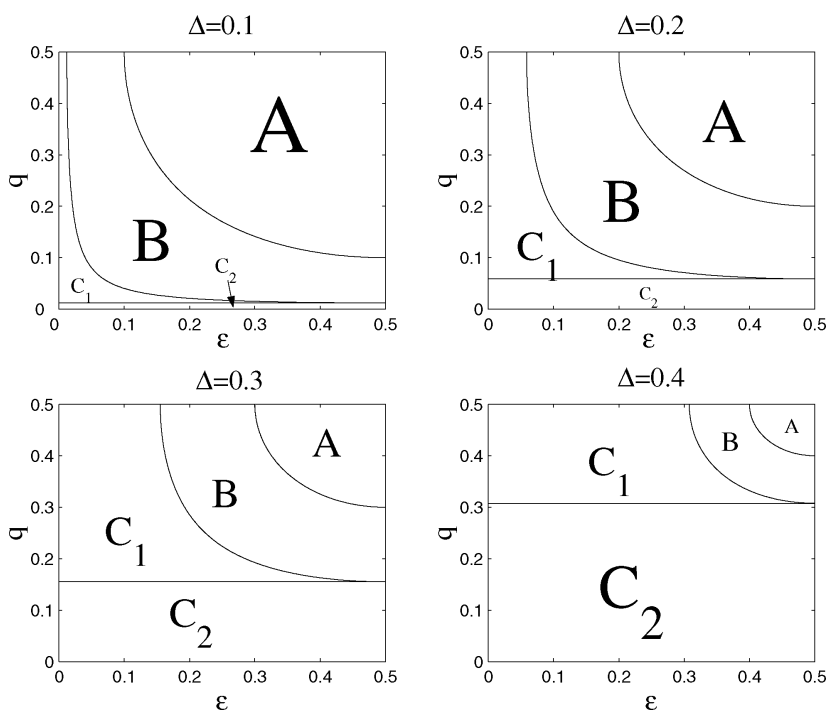

Fig. 8. The regions for the $(\varepsilon, q)$ pairs in the binary DMS $\{q, 1-q\}$ and BSC $(\varepsilon)$ system of Example 5 with Hamming distortion for different values of the distortion threshold $\Delta$ with $t=1$. Note that $E_{J}^{\Delta}=0$ on the boundary between $\boldsymbol{A}$ and $\boldsymbol{B}$, and $E_{\vec{J}}^{\Delta}>0$ is determined on the boundary between $\boldsymbol{B}$ and $\boldsymbol{C}_{1}$.

different distortion thresholds. We fix the BSC parameter $\varepsilon=$ 0.2 , and vary $q$ from 0 to 0.5 . In Fig. 9, Segment (or Section) 1 is determined by (66), and Segments 2 and 3 are determined by (65). Furthermore, the lower bound coincides with the upper bound (67) in Segment 3; i.e., the JSCC exponent is exactly determined in Segment 3.

\section{CONCLUSION}

In this work, we establish equivalent parametric representations of Csiszár's lower and upper bounds for the JSCC exponent $E_{J}$ of a communication system with a DMS and a DMC, and we obtain explicit conditions for which the JSCC exponent is exactly determined. As a result, the computation of the bounds for $E_{J}$ is facilitated for arbitrary DMS-DMC pairs. Furthermore, the bounds enjoy closed-form expressions when the channel is symmetric. A byproduct of our result is the fact that Csiszár's random-coding lower bound for $E_{J}$ is in general larger than Gallager's lower bound [23].

We also provide a systematic comparison between $E_{J}$ and $E_{T}$, the tandem coding error exponent. We show that JSCC can at most double the error exponent vis-a-vis tandem coding by proving that $E_{J} \leq 2 E_{T}$ and we provide the condition for achieving this doubling effect. In the case where this upper bound is not tight, we also establish sufficient explicit conditions under which $E_{J}>E_{T}$. Numerical results indicate that $E_{J} \approx 2 E_{T}$ for a large class of DMS-DMC pairs, hence illustrating the substantial potential benefit of JSCC over tandem coding. This benefit is also shown to result into a power saving gain of more than $2 \mathrm{~dB}$ for a binary DMS and a BPSK-modulated AWGN/Rayleigh channel with finite output quantization. Finally, we partially investigate the computation of Csiszár's lower and upper bounds for the lossy JSCC exponent underthe Hamming distortion measure, and obtain equivalent representations for these bounds using the same approach as for the lossless JSCC exponent. 


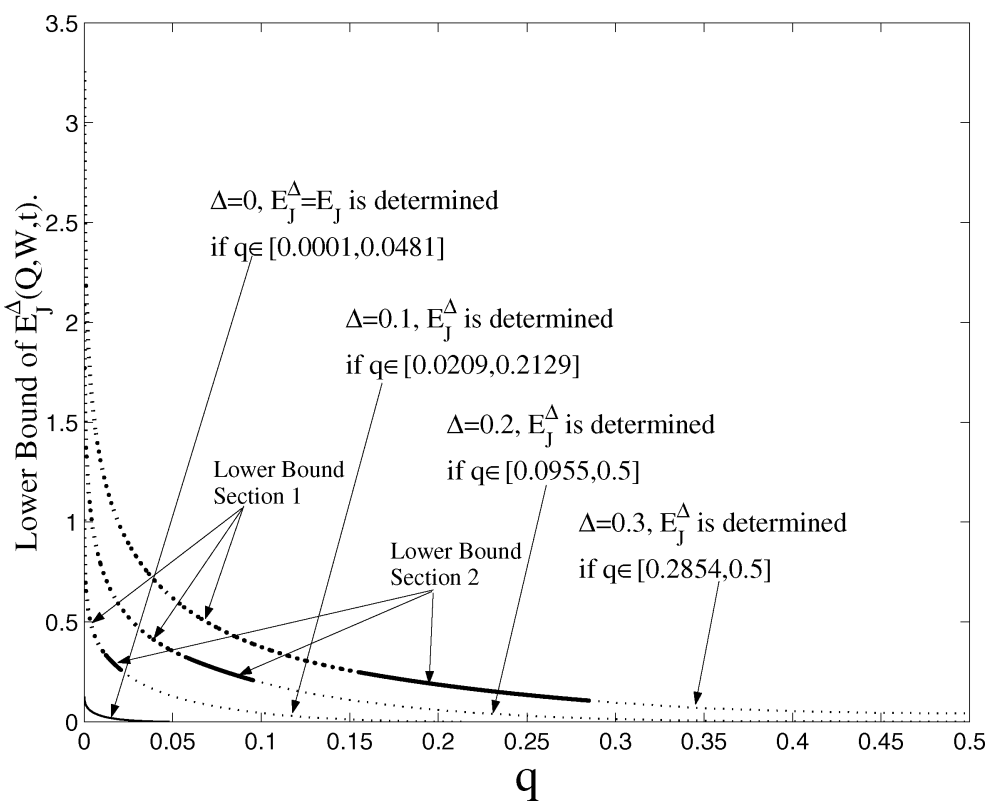

Fig. 9. Fix $\varepsilon=0.2$. The JSCC exponent lower bound of the binary DMS $\{q, 1-q\}(q \leq 0.5)$ and BSC $(\varepsilon)$ pairs under Hamming distortion with $t=1$. For $\Delta=0, E_{J}^{\Delta}$ is determined if $q \in[0.0001,0.0481]$, which is the same as the random-coding lower bound for the lossless JSCC error exponent. For $\Delta=0.1, E_{J}^{\Delta}$ is determined if $q \in[0.0209,0.2129]$. For $\Delta=0.2, E_{J}^{\Delta}$ is determined if $q \in[0.0955,0.5]$. For $\Delta=0.3, E_{J}^{\Delta}$ is determined if $q \in[0.2854,0.5]$.

\section{APPENDIX A}

\section{PROOF OF LEMMA 6}

Recall that the rate-distortion function $R(Q, \Delta)$ for a binary DMS $Q=\{q, 1-q\}$ under the Hamming distortion measure is given by (e.g., [16])

$$
R(Q, \Delta)= \begin{cases}h_{b}(q)-h_{b}(\Delta), & 0 \leq \Delta \leq q \\ 0, & \Delta>q\end{cases}
$$

Clearly, $F(R, Q, \Delta)=0$ for $R \leq 0$ since the infimum in (60) is attained at $P=Q$. Similarly, since $R(P, \Delta) \leq 1-h_{b}(\Delta)$ for all $P, F(R, Q, \Delta)=\infty$ for $R>1-h_{b}(\Delta)$. For the remainder of the proof, we assume $0<R \leq 1-h_{b}(\Delta)$.

1) Case of $0 \leq \Delta \leq q$. For $R \leq R(Q, \Delta)=h_{b}(q)-h_{b}(\Delta)$, we have

$$
\begin{aligned}
& F(R, Q, \Delta) \\
& \quad=\inf _{P: R(P, \Delta)>R} D(P \| Q)=\left.D(P \| Q)\right|_{P=Q}=0 .
\end{aligned}
$$

For $h_{b}(q)-h_{b}(\Delta)<R \leq 1-h_{b}(\Delta)$, we have

$$
\begin{aligned}
F(R, Q, \Delta)= & \inf _{P: R(P, \Delta)>R} D(P \| Q) \\
= & \min _{P \triangleq\{p, 1-p\}: R(P, \Delta)=R} D(P \| Q) \\
= & \min _{p: h_{b}(p)-h_{b}(\Delta)=R} D(P \| Q) \\
= & e\left(R+h_{b}(\Delta), Q\right), \\
& \text { for } H(Q) \leq R+h_{b}(\Delta) \leq \log |\mathcal{S}| \\
= & \sup _{\rho \geq 0}\left[\rho\left(R+h_{b}(\Delta)\right)-E_{s}(\rho)\right] \\
= & \sup _{\rho \geq 0}\left[\rho R-E_{s}^{\Delta}(\rho, Q)\right] .
\end{aligned}
$$

Here (70) follows from the facts that the continuous function

$$
\theta(p) \triangleq p \log \frac{p}{q}+(1-p) \log \frac{1-p}{1-q}
$$

is increasing for $p \geq q$ and $R(P, \Delta)$ given in (69) is continuous and increasing in $p$ for $\Delta \leq p \leq \frac{1}{2}$. In (71), we note that $H(Q)=h_{b}(q)$ and that $\log |\mathcal{S}|=1$ as the source is binary. Equation (72) follows by the well-known parametric form of source exponent function introduced by Blahut [13] and noting that

$$
R^{\prime} \triangleq R+h_{b}(\Delta) \in[H(Q), \log |\mathcal{S}|] .
$$

2) Case of $\Delta>q$. For $0<R \leq 1-h_{b}(\Delta)$, similarly as (71), we have

$$
F(R, Q, \Delta)=e\left(R^{\prime}, Q\right)=\sup _{\rho \in A}\left[\rho R^{\prime}-E_{s}(\rho)\right]
$$

where $R^{\prime}=R+h_{b}(\Delta)$ such that

$$
H(Q)<h_{b}(\Delta)<R^{\prime} \leq 1=\log |\mathcal{S}|
$$

and

$$
\begin{aligned}
A & =\left\{\rho^{*}:\left.\frac{\partial\left[\rho R^{\prime}-E_{s}(\rho)\right]}{\partial \rho}\right|_{\rho=\rho^{*}}=0, h_{b}(\Delta) \leq R^{\prime} \leq 1\right\} \\
& =\left\{\rho^{*}: h_{b}(\Delta) \leq R^{\prime}=H\left(Q^{\left(\rho^{*}\right)}\right) \leq 1\right\} \\
& =\left\{\rho^{*}: \rho_{0} \leq \rho^{*}<\infty\right\}
\end{aligned}
$$

where $\rho_{0}$ is the unique root of equation $H\left(Q^{(\rho)}\right)=h_{b}(\Delta)$ and $\rho_{0}>0$. Here (73) follows from the monotone property of $H\left(Q^{(\rho)}\right)$. Therefore, we write

$$
F(R, Q, \Delta)=\sup _{\rho \geq \rho_{0}}\left[\rho R-E_{s}^{\Delta}(\rho, Q)\right] .
$$

In fact, it can be shown that $\rho_{0}$ is the right-slope of $F(R, Q, \Delta)$ at $R=R(Q, \Delta)$. 


\section{REFERENCES}

[1] R. Ahlswede, "Extremal properties of rate-distortion functions," IEEE Trans. Inf. Theory, vol. 36, no. 1, pp. 166-171, Jan. 1990.

[2] F. Alajaji, N. Phamdo, N. Farvardin, and T. Fuja, "Detection of binary Markov sources over channels with additive Markov noise," IEEE Trans. Inf. Theory, vol. 42, no. 1, pp. 230-239, Jan. 1996.

[3] F. Alajaji, N. Phamdo, and T. Fuja, "Channel codes that exploit the residual redundancy in CELP-encoded speech," IEEE Trans. Speech Audio Process., vol. 4, no. 5, pp. 325-336, Sep. 1996.

[4] F. Alajaji and N. Phamdo, "Soft-decision COVQ for Rayleigh-fading channels," IEEE Commun. Lett., vol. 2, no. 6, pp. 162-164, Jun. 1998.

[5] E. Arikan and N. Merhav, "Guessing subject to distortion," IEEE Trans. Inf. Theory, vol. 44, no. 3, pp. 1041-1056, May 1998.

[6] - "Joint source-channel coding and guessing with application to sequential decoding," IEEE Trans. Inf. Theory, vol. 44, no. 5, pp. 1756-1769, Sep. 1998.

[7] S. Arimoto, "'On the converse to the coding theorem for discrete memoryless channels," IEEE Trans. Inf. Theory, vol. IT-19, no. 3, pp. 357-359, May 1973.

[8] - "Computation of random coding exponent functions," IEEE Trans. Inf. Theory, vol. IT-22, no. 6, pp. 665-671, Nov. 1976.

[9] E. Ayanoglu and R. Gray, "The design of joint source and channel trellis waveform coders," IEEE Trans. Inf. Theory, vol. IT-33, no. 6, pp. 855-865, Nov. 1987.

[10] V. B. Balakirsky, "Joint source-channel coding with variable length codes," Probl. Inform. Transm., vol. 1, no. 37, pp. 10-23, Jan./Mar. 2001.

[11] A. Barg and A. McGregor, "Distance distribution of binary codes and the error probability of decoding," IEEE Trans. Inf. Theory, vol. 51, no. 12, pp. 4237-4246, Dec. 2005.

[12] D. P. Bertsekas, A. Nedić, and A. E. Ozdagler, Convex Analysis and Optimization. Belmont, MA: Athena Scientific, 2003.

[13] R. E. Blahut, "Hypothesis testing and information theory," IEEE Trans. Inf. Theory, vol. IT-20, no. 4, pp. 405-417, Jul. 1974.

[14] B. D. Bunday, Basic Optimization Methods. London, U.K.: Arnold, 1984.

[15] P.-N. Chen and F. Alajaji, "Optimistic Shannon coding theorems for arbitrary single-user systems," IEEE Trans. Inf. Theory, vol. 45, no. 7, pp. 2623-2629, Nov. 1999.

[16] T. M. Cover and J. A. Thomas, Elements of Information Theory. New York: Wiley, 1991.

[17] I. Csiszár, "Joint source-channel error exponent," Probl. Contr. Inf. Theory, vol. 9, pp. 315-328, 1980.

[18] —, "On the error exponent of source-channel transmission with a distortion threshold," IEEE Trans. Inf. Theory, vol. IT-28, no. 6, pp. 823-828, Nov. 1982

[19] I. Csiszár and J. Körner, Information Theory: Coding Theorems for Discrete Memoryless Systems. New York: Academic, 1981.

[20] J. G. Dunham and R. M. Gray, "Joint source and noisy channel trellis encoding," IEEE Trans. Inf. Theory, vol. IT-27, no. 4, pp. 516-519, Jul. 1981.

[21] N. Farvardin, "A study of vector quantization for noisy channels," IEEE Trans. Inf. Theory, vol. 36, no. 4, pp. 799-809, Jul. 1990.

[22] T. Fine, "Properties of an optimum digital system and applications," IEEE Trans. Inf. Theory, vol. IT-10, no. 4, pp. 443-457, Oct. 1964.

[23] R. G. Gallager, Information Theory and Reliable Communication. New York: Wiley, 1968.

[24] M. Gastpar, B. Rimoldi, and M. Vetterli, "To code, or not to code: Lossy source-channel communication revisited," IEEE Trans. Inf. Theory, vol. 49, no. 5, pp. 1147-1158, May 2003.

[25] J. D. Gibson and T. R. Fisher, "Alphabet-constrained data compression," IEEE Trans. Inf. Theory, vol. IT-28, no. 3, pp. 443-457, May 1982.

[26] R. M. Gray and D. S. Ornstein, "Sliding-block joint source/noisychannel coding theorems," IEEE Trans. Inf. Theory, vol. IT-22, no. 6, pp. 682-690, Nov. 1976.

[27] J. Hagenauer, "Source-controlled channel decoding," IEEE Trans. Commun., vol. 43, no. 9, pp. 2449-2457, Sep. 1995.

[28] T. S. Han, Information-Spectrum Methods in Information Theory. New York: Springer-Verlag, 2003.

[29] M. E. Hellman, "Convolutional source encoding," IEEE Trans. Inf. Theory, vol. IT-21, no. 6, pp. 651-656, Nov. 1975.

[30] B. Hochwald and K. Zeger, "Tradeoff between source and channel coding," IEEE Trans. Infm. Theory, vol. 43, no. 5, pp. 1412-1424, Sep. 1997.

[31] F. Jelinek, Probabilistic Information Theory. New York: McGrawHill, 1968 .
[32] V. N. Koshelev, "Direct sequential encoding and decoding for discrete sources," IEEE Trans. Inf. Theory, vol. IT-19, no. 3, pp. 340-343, May 1973.

[33] H. Kumazawa, M. Kasahara, and T. Namekawa, "A construction of vector quantizers for noisy channels," Electron. Eng. Jpn., vol. 67-B, no. 4, pp. 39-47, 1984.

[34] A. Kurtenbach and P. Wintz, "Quantizing for noisy channels," IEEE Trans. Commun. Technol., vol. COM-17, no. 4, pp. 291-302, Apr. 1969.

[35] J. Lim and D. L. Neuhoff, "Joint and tandem source-channel coding with complexity and delay constraints," IEEE Trans. Commun., vol. 51, no. 5, pp. 757-766, May 2003.

[36] D. G. Luenberger, Optimization by Vector Space Methods. New York: Wiley, 1969.

[37] K. Marton, "Error exponent for source coding with a fidelity criterion," IEEE Trans. Inf. Theory, vol. IT-20, no. 3, pp. 197-199, Mar. 1974.

[38] J. L. Massey, "Joint source and channel coding," in Communications and Random Process Theory, J. K. Skwirzynski, Ed, The Netherlands: Sijthoff and Nordhoff, 1978, pp. 279-293.

[39] D. Miller and K. Rose, "Combined source-channel vector quantization using deterministic annealing," IEEE Trans. Commun., vol. 42, no. 2/3/4, pp. 347-356, Feb./Mar./Apr. 1994.

[40] J. W. Modestino and D. G. Daut, "Combined source-channel coding of images," IEEE Trans. Commun., vol. COM-27, no. 11, pp. 1644-1659, Nov. 1979.

[41] N. Phamdo and F. Alajaji, "Soft-decision demodulation design for COVQ over white, colored, and ISI Gaussian channels," IEEE Trans. Commun., vol. 46, no. 9, pp. 1499-1506, Sep. 2000.

[42] R. T. Rockafellar, Conjugate Duality and Optimization. Philadelphia, PA: SIAM, 1974.

[43] H. L. Royden, Real Analysis, 3rd ed. New York: Macmillan, 1988.

[44] K. Sayood and J. C. Borkenhagen, "Use of residual redundancy in the design of joint source/channel coders," IEEE Trans. Commun., vol. 39, no. 6, pp. 838-846, Jun. 1991.

[45] C. E. Shannon, "A mathematical theory of communication," Bell Syst. Tech. J., vol. 27, pp. 379-423, Jul./Oct. 1948.

[46] S. Shamai (Shitz), S. Verdú, and R. Zamir, "Systematic lossy source/channel coding," IEEE Trans. Inf. Theory, vol. 44, no. 2, pp. 564-579, Mar. 1998.

[47] M. Skoglund and P. Hedelin, "Hadamard-based soft decoding for vector quantization over noisy channels," IEEE Trans. Inf. Theory, vol. 45, no. 2, pp. 515-532, Mar. 1999

[48] M. Skoglund, "Soft decoding for vector quantization over noisy channels with memory," IEEE Trans. Inf. Theory, vol. 45, no. 3, pp. 1293-1307, May 1999.

[49] G. Taricco, "On the capacity of the binary input Gaussian and Rayleigh fading channels," Europ. Trans. Telecommun., vol. 7, no. 2, Mar./Apr. 1996.

[50] V. A. Vaishampayan and N. Farvardin, "Joint design of block source codes and modulation signal sets," IEEE Trans. Inf. Theory, vol. 38, no. 4, pp. 1230-1248, Jul. 1992.

[51] S. Vembu, S. Verdú, and Y. Steinberg, "The source-channel separation theorem revisited," IEEE Trans. Inf. Theory, vol. 41, no. 1, pp. 44-54, Jan. 1995

[52] A. J. Viterbi and J. K. Omura, Principles of Digital Communication and Coding. New York: McGraw-Hill, 1979.

[53] T. Weissman, E. Ordentlich, G. Seroussi, S. Verdú, and M. J. Weinberger, "Universal discrete denoising: Known channel," IEEE Trans. Inf. Theory, vol. 51, no. 1, pp. 5-28, Jan. 2005.

[54] K. A. Zeger and A. Gersho, "Pseudo-Gray coding," IEEE Trans. Commun., vol. 38, no. 12, pp. 2147-2158, Dec. 1990.

[55] Y. Zhong, F. Alajaji, and L. L. Campbell, "When is joint source-channel coding worthwhile: An information theoretic perspective," in Proc. 22nd Bienn. Symp. Communications, Kingston, ON, Canada, Jun. 2004, pp. $121-123$.

[56] _ "On the computation of the joint source-channel error exponent for memoryless systems," in Proc. 2004 IEEE Int. Symp. Information Theory, Chicago, IL, Jun./Jul. 2004, p. 477.

[57] _ (2005) "On the Joint Source-Channel Coding Error Exponent for Discrete Memoryless Systems: Computation and Comparison with Separate Coding," Mathematics and Engineering Tech. Rep.. Dept. Math. and Statist., Queen's Univ., Kingston, ON, Canada. [Online]. Available: http://markov.mast.queensu.ca/publications.html/ and http://arxiv.org/abs/cs.IT/0601006

[58] G.-C. Zhu, F. Alajaji, J. Bajcsy, and P. Mitran, "Transmission of nonuniform memoryless sources via nonsystematic turbo codes," IEEE Trans. Commun., vol. 52, no. 8, pp. 1344-1354, Aug. 2004. 